\title{
Method for fabrication and verification of conjugated nanoparticle-antibody tuning elements for multiplexed electrochemical biosensors.
}

Jeffrey T. La Belle, Ph.D. ${ }^{a, b,{ }^{*}}$; Aaron Fairchild, M.S. ${ }^{a, b}$; Ugur K. Demirok, Ph.D. ${ }^{\text {; }}$; Aman Verma, M.S. ${ }^{a, b}$ Author Affiliations: ${ }^{a}$ Harrington Program of Biomedical Engineering, in the School of Biological and Health Systems Engineering, and ${ }^{b}$ Biodesign Institute, Arizona State University, Tempe 85287, AZ, USA; Arizona State University, Tempe 85287, AZ, USA.

Keywords: biosensor, health management, disease monitoring, electrochemical impedance spectroscopy

*Corresponding Author: Jeffrey T. La Belle, Ph.D., 550 East Orange St., P.O. Box 879709, Tempe, AZ 9709; email address jeffrey.labelle@asu.edu

\begin{abstract}
There is a critical need for more accurate, highly sensitive and specific assay for disease diagnosis and management. A novel, multiplexed, single sensor using rapid and label free electrochemical impedance spectroscopy tuning method has been developed. The key challenges while monitoring multiple targets is frequency overlap. Here we describe the methods to circumvent the overlap, tune by use of nanoparticle (NP) and discuss the various fabrication and characterization methods to develop this technique. First sensors were fabricated using printed circuit board (PCB) technology and nickel and gold layers were electrodeposited onto the PCB sensors. An off-chip conjugation of gold NP's to molecular recognition elements (with verification technique) is described as well. A standard covalent immobilization of the molecular recognition elements is also discussed with quality control techniques. Finally use and verification of sensitivity and specificity is also presented. By use of gold NP's of various sizes, we have demonstrated the possibility and shown little loss of sensitivity and specificity in the molecular recognition of inflammatory markers as "model" targets for our tuning system. By selection of other sized NP's or NP's of various materials, the tuning effect can be further exploited. The novel platform technology developed could be utilized in critical care, clinical management and at home health and disease management.
\end{abstract}

\section{Introduction}

With the advent and interest in genomics (Tsirigos, 2011), proteomics (Weston, 2004), metabolomics (Ala-Korpela, 2012), vaccine development (Bensi, 2012), and personalized medicine (Workman, 2012), high throughput systems are of great desire (Stanley, 2012). However, many of these technologies are time consuming, labor intensive, have issues with lower limits of detection or multiplex factor, or require the use of special reagents and sample preparation or labels on the target(s) of interest. Typical modes of detection are optical based (Choi, 2012) or electrochemical in nature (Mu, 2012). 
Likewise due to the variety of techniques, platforms, and sensors there are many means to assemble these systems to do the high throughput multiplexed assays. One common method, utilized in many industries for mass fabrication is printed circuit board design (Rabinovich, 2012). Here, typically a fiberglass substrate is copper-clad, then coated with photoresist which protects the copper in subsequent steps. The pattern can be negatively or positively developed in the photoresist. The excess copper is removed leaving behind circuitry, leads and/or sensors in copper. Ideal substrate layers, such as gold or other metals can then be deposited on via an electrodeless process (Li, 2012) or electrodeposited (Liu, 2012) onto the copper or other interface layers. The deposition of gold allows for immobilization of molecular recognition elements such as antibodies using covalent attachment methods (La Belle, 2007). Basically, a long carbon chain alkanethiol can self-assemble via thiol linkage to the hydroxyl group on the gold surface. Through the use of zero-crosslength linkers such as sulfo-NHydroxysuccinimid (sulfo-NHS) and ethyl(dimethylaminopropyl) carbodiimide (EDC). Then the molecular recognition elements are added and the sensors are ready for testing.

A common method of electrochemical testing begins with a cyclic voltammogram (CV) in order to determine the formal potential of the electrochemical cell or sensor. This determines the DC offset for the AC sweep used in electrochemical impedance spectroscopy (EIS) based techniques. Next, a common means to validate sensor performance is to run a concentration gradient or scale against the sensors to determine many typical parameters such as: lower limits of detection (LLD), upper limits of detection (ULD), from these one obtains a dynamic range. Other parameters include responsivity (slope of the gradient), reproducibility (standard deviation divided by the average in percent), tightness of fit ( $r$ square of trend line applied and behavior (liner, non-linear, etc). One unique parameter in EIS is optimal binding frequency, which can be determined across the frequency spectra of the gradient by looking for best $r$-square and then responsivity. This parameter identifies when optimal detection of antigenantibody recognition occurred.

However, herein lies the challenge with high throughput marker evaluation, the frequency spectra used ranges from $1 \mathrm{~Hz}$ to $100 \mathrm{kHz}$ with most of the activity measured between 1 to $1,000 \mathrm{~Hz}$. There is much room for overlap. With this apparent limitation, single sensor, multimarker, label-free detection seemed difficult to overcome, until an idea to tune the frequency spectra arose (La Belle, 2011). By addition of metal nanoparticles (NP's) to the molecular recognition elements, it might be possible to tune the optimal frequencies of two targets away from one another if functionality still remained. With the developed and discussed technique herein, a solution has been found, developed, and modeled. The future of having a single sensor, multiplexed sensor system for personalized medicine is one step closer.

\section{Method of Sensor Fabrication}

\subsection{Printed Circuit Board Array Fabrication}

The substrate for this sensor array is the common printed circuit board (PCB) used commonly in electronics. The first step in fabrication is design. Using common computer aided drafting (CAD) software, such as AutoCAD (Autodesk, Inc, San Rafael, CA, USA) using dimension-based design one can 
draw the electrical connection leads, lines to the electrodes and of course, the reference electrode $(\mathrm{RE})$, counter electrode (CE), and working electrode (WE) for one sensor. Making the array is simply copy/paste one sensor to the next sport and continue until all sensors are complete (Fig. $1 \mathrm{~A}$ ). The resulting design (called the mask at this point), once printed onto a transparency can be used in a photofabrication (photolithography) step using a pre-sensitized (has a layer of photoresist already on it) copper clad PCB board, 6" $\times 6^{\prime \prime}$ is commonly available and can fit many arrays onto one board thus increasing efficiency (Circuit Specialists, Mesa AZ, USA). Once printed, the resulting array would be comprised of the aforementioned substrate in Fig. 1A (a), the electrical leads for connect ion to an electrochemical analyzer in Fig. $1 \mathrm{~A}(\mathrm{~b})$, the wires running between the leads and electrodes in Fig. $1 \mathrm{~A}$ (c), and of course, the electrochemical electrodes, RE in Fig. 1A (d), WE in Fig. 1A (e), and CE in Fig. 1A (f). Once fabrication is completed, the final goal would be to have a gold layer on top, that would allow for surface chemistries to be performed to immobilize molecular recognition in later steps. To end up with gold, an interface layer must first be placed onto the copper, for which nickel is commonly used. As seen in the multi-layer (side view) schematic (Fig. 1B). The gold layer in Fig. 1B (a) is deposited on top of the nickel layer Fig. 1B (b) which was deposited on top of the copper in Fig. $1 \mathrm{~B}$ (c) on the fiberglass PCB layer in Fig. 1B (d).

However, before the layers can be deposited, the PCB array must translate from CAD to the PCB. In order to do this, the CAD must be printed out onto standard mylar transparencies available at any office supply store. The mask is then placed no top of the pre-sensitized copper clad board into a UV light box (Circuit Specialists, Mesa AZ, USA) and exposed for 2 min. The exposed photoresist is removed in a bath of pH 14.0 sodium hydroxide developer (Circuit Specialists, Mesa AZ, USA) for 2 min, with the excess developer is rinsed off using distilled water thereby stopping the chemical reaction. Next the board is now placed into a bubbling water tank containing a $\mathrm{pH} 1.0$ solution of ammonium persulfate (Circuit Specialists, Mesa AZ, USA) to remove the unprotected copper that was exposed after the photoresist was removed in the previous step leaving a series of sensors or arrays ready for electroplating as in Fig. 2 (a-f). Next the sensor arrays are separated from one another typically using a band saw or table saw (MicroMark, Berkeley Heights NJ, USA). Once these arrays have been separated, it is best to store them such that the copper-photoresist side are not damaged or scratched.

\subsection{Electroplating}

Electrochemical deposition is one of the major techniques for bottom-up synthesis of electronics. It is usually performed in aqueous solutions at ambient conditions, involves simple instrumentation and operation, and is versatile and cost effective. Alongside its many advantages, electrodeposition has a key drawback. The deposited material has to be electrically conductive to sustain the growth after initial oxidation/reduction of the target species on the electrode. Although this issue prohibits fabrication of insulators by this method, a large variety of materials such as metals (Zeng, 2000; Davydov, 1999; Iwasaki, 1999; Ferre, 1997; Li, 2001; Haddon, 2002), alloys (Saedi, 2005), semiconductors (Ferain, 1993), superconductors (Sloan, 2002), and conducting polymers (Wang, 1998) can be deposited electrochemically. Electrochemical deposition, in general, is performed by application of an electrical field to a solution containing the ions of a target species. Electricallyenhanced diffusion of a reactive ion towards the deposition site (i.e., electrode) is followed by an 
electron transfer from or to the ion, resulting in oxidative or reductive deposition of the desired material onto the electrode, respectively.

The process of electroplating begins with a setup (Fig. 3) whereby a bath of a solution of metals ions has an anode and cathode. The anode is a metal made from the same material in the bath (the same as the desired plated layer). The substrate that will have this material plated on it sits within a fixed distance from the anode and acts as the cathode. A power supply is used to drive the reaction. A holder apparatus that can keep the distance between anode and cathode fixed and hold all parts immobile is also desirable and can be readily made from high density polyethylene and nylon screws (U.S. Plastic Corp., Lima, Ohio USA).

Begin by preparing the degreaser solution. Make $500 \mathrm{ml}$ of fresh degreaser, by $59.9132 \mathrm{~g} / \mathrm{L}$ of distilled water (Caswell Lyons NY, USA). Place the beaker on a hot plate (VWR International, Radnor, PA USA) and bring to $93.3^{\circ} \mathrm{C}$ for $2.5 \mathrm{~min}$. Make fresh nickel solution by heating $500 \mathrm{ml}$ distilled water to $43.3^{\circ} \mathrm{C}$ using a hot plate and a stirrer. Add in $119.8264 \mathrm{~g}$ Nickel solution powder. Stir with no major agitation until all of the nickel power has dissolved. Test the $\mathrm{pH}$ at temperature and at room temperature. Add 20 $\mathrm{ml}$ of brightener to the nickel solution. The nickel solution (Caswell Lyons NY, USA) is then poured into the crystallization dish that is in the water bath. Fill to cover the electrode array to appropriate height (last the electrode wires and electrodes basically). Heat up the water bath (VWR International, Radnor, PA USA) by pressing the "on" button located at the left side of the back of the water bath machine. It should already be set for $68^{\circ} \mathrm{C}$. It takes an hour to heat up. Fill a $500 \mathrm{ml}$ beaker with distilled water and place on hot plate with a lid to prevent evaporation and for faster heating to refresh solutions. Set up Nickel plating circuit using the constant current power supply (Circuit Specialists, Mesa AZ, USA) by setting the digital/manual power supply for $0.031 \mathrm{~A}$ at $2.0 \mathrm{v}$ and place nickel anode in the solution sitting as low as it can in the slot. [turn unit on - vset - 2 (volt) - vset - esc - iset - 29(mA) - iset - esc]. An EXCEL template was generated to compute the amount of charge and thereby the amount of ions of metals being deposited onto the electrodes.

Next step, cleaning the electrodes in the degreaser, begin by rising the PCB's with acetone (Sigma Aldrich, St. Louis MO, USA). Allow to air dry. Using a strainer or tweezers, agitate electrode in heated degreaser for $90 \mathrm{sec}$. Immediately move the electrode to the water that is being heated on the hot plate and agitate for $45 \mathrm{sec}$ or until software deems enough charge has been plated. Use a Kimwipe (VWR International, Radnor, PA USA) to dry. Immediately move to nickel plating. Put the dry electrode into the edge connector (Digikey Electronics, Thief River Falls, MN USA). Make sure the edge connector is hooked up to the cable that is hooked directly to the power supply. The nickel anode should be connected to the cable that is hooked up to the digital multimeter (Digikey Electronics, Thief River Falls, MN USA). The meter should be turned on and set to 40mA. Place the electrode/edge connector in the solution, resting in the notch. When everything is set up, simultaneously start the power supply and the stop watch. Record data from meter every $60 \mathrm{sec}$ from the EXCEL file which will calculate the time left in solution. When time is up, turn the current from the power supply off. Take the connector and electrode out of the solution and carefully rinse only the bottom of the electrode with DI water. Then place the connector and electrode in the gold plating solution. Switch the cable from the nickel anode to 
the gold anode. Change the power supply to $13 \mathrm{~mA}$ and add the $100 \mathrm{kOhm}$ resistor to provide voltage division. Follow the same procedure as the nickel plating, recording the data every 30 seconds. After the gold plating process is completed, remove the connector and the electrode from the solution then disassemble the electrode from the edge connector. Rinse the electrode well with DI water. This concludes the nickel and gold plating process. Electrodes can be stored dry (under nitrogen) or aqueous to prevent contamination of the gold surface.

\section{Method of Molecular Recognition Immobilization}

Immobilization is the technique for the fixation of biological components (i.e. cells, antibodies, enzymes) on a solid surface (McNaught, 1997). In order to ensure proper functionality of the biosensor (Fig. 4), appropriate immobilization protocols and techniques must be employed. For gold disk electrodes ( $\mathrm{CH}$ Instruments, Austin TX, USA), each electrode is polished 120 times sequentially on alumina pads that are 3,1 , and 0.05 micron-sized. The electrodes are then thoroughly rinsed with distilled water and subsequently sonicated in ethanol for 15 minutes, followed by distilled water for an additional 15 minutes. After polishing and sonication, the electrodes are analyzed using CV, and EIS is performed using the adjusted formal potential after the electrode has been polished. If the electrodes still show an appreciable surface resistance, the polishing and sonication steps are repeated. This is done to ensure that the electrode surface has the least amount of surface resistance possible, which is indicative of having a clean immobilization surface.

After the electrodes are cleaned satisfactorily, they are immersed in a $10 \mathrm{mM}$ solution of 16-MHDA in ethanol for one hour in order to form alkane self-assembled monolayers (SAMs). The concentration of MHDA used to form the SAMs can be adjusted depending on the incubation time available, but should be of a concentration that is appropriate to sufficiently cover the electrode surface. After gently rinsing with distilled water, the electrodes are immersed in an $80 \mathrm{mM}$ solution of EDC/NHS for one hour in order to facilitate the conversion of the carboxylic acid group of the alkane monolayer to an amine group for subsequent antibody attachment. After gently rinsing with phosphate buffered saline (PBS), the electrodes are subsequently immersed in a solution of $25 \mu \mathrm{g} / \mathrm{mL}$ solution of $\alpha$-IL-12 antibody for an additional hour, rinsed again gently with PBS, and then immersed in $1 \%$ solution of ethanolamine for one half hour for blocking. The electrodes are then stored dry at $4^{\circ} \mathrm{C}$ until further use with actual antigen samples.

\section{Method of Electrochemical Techniques and Optimal Frequency Detection}

\subsection{Cyclic Voltammetry}

Cyclic voltammetry (CV) is a variant of the linear sweep voltammetry (LSV) that is effective and versatile in mechanistic studies of electrochemical systems. Due to its provision of many basic components of electrochemical reactions, simplicity, and efficiency, it is usually the starting method in electrochemical characterization of novel systems. A CV experiment starts at the initial or lower potential $\left(E_{1}\right)$ and is then ramped up towards its upper target $\left(E_{2}\right)$. After the initial non-faradaic region, the neutral species starts to get oxidized as the potential becomes more positive. As oxidization occurs 
on the electrode, the mass transfer rate of neutral species towards the electrode increases, and so does the current. This current reaches a maximum peak, after which the surface concentration of the neutral species starts to decline due to depletion from the solution, resulting in a reduced oxidation current. The process is then repeated in the same manner for the reverse scan to obtain the reduction behavior. The potential cycle may be repeated to obtain additional information with system stability typically observed after the third full sweep as these types of successive measurements can prove useful especially for the reversibility and stability studies. The step, or scan rate, at which the potential is varied can also be used to extract further information pertaining to mass transfer profile near the electrode as the thickness of the diffusion layer can be adjusted by the scan rate.

Theoretical aspects of CV are well studied. In a system where the electron transfer occurs much faster than the diffusion of an electroactive species to the electrode, the peak current, Ip, can be calculated from the Randles-Sevcik relationship as follows (Bard, 2000):

$$
I_{P}=k n^{3 / 2} A D^{1 / 2} C v^{1 / 2}
$$

where $\mathrm{k}$ is a constant, $\mathrm{n}$ is the number of moles of electrons transferred, $\mathrm{A}$ is the area of the electrode, $D$ is the diffusion coefficient, $C$ is the solution concentration and $v$ is the scan rate. This relationship can be used to determine if a reaction is diffusion limited by simply plotting the peak current versus the square root of the scan rate. If a linear plot is obtained then the reaction is mass transfer limited. Moreover, the peak potentials at varying scan rates can be compared to find out if a reaction is reversible. In a reversible reaction where the electron transfer rate is fast, all peaks should appear at the same potential at different scan rates. Formal potential $\left(E_{0}\right)$ of the redox reaction and the diffusion layer thickness can also be obtained from a CV. As such, $E_{0}$ can be found at the value that corresponds to $85 \%$ of the peak potential and the thickness of the diffusion layer, $\delta$, can be estimated from $(D t)^{1 / 2}$, where $D$ is the diffusion coefficient and $t$ is time in seconds. Thus, cyclic voltammetry is very rich in information and is one of the very powerful techniques in the armory of electrical characterization tools.

The application of CV is two-fold here. First it can be used as a quality control to determine if the electrodes are cleaned properly, as the CV for a good, clean bare gold electrode has high current values and near perfect shape. Secondly, the CV can be used to determine the formal potential for the system as well, that is, the halfway point between oxidization and reduction peaks. Basically, the electrodes are connected to a $\mathrm{CHI} 660 \mathrm{C}$ Impedance Analyzer, run in $\mathrm{CV}$ mode from $-1.0 \mathrm{~V}$ to $+1.0 \mathrm{~V}$ sweeps, three full scans. Scan rate was typically $0.1 \mathrm{~V} / \mathrm{s}$ with a resolution set at $0.01 \mathrm{~A} / \mathrm{V}$. A redox probe solution of $5 \mathrm{mM}$ ferricyanide: $5 \mathrm{mM}$ ferrocyanide (Sigma Aldrich, St. Louis MO, USA) in $10 \mathrm{mM}$ Phosphate Buffered Saline (PBS) at pH 7.4 (VWR International, Radnor, PA USA) henceforth called redox probe was used in all electrochemical testing unless noted otherwise. Peaks are measured and averaged to determine Formal Potential.

\subsection{Electrochemical Impedance Spectroscopy}

Originally developed by Sluyters et. al., electrochemical impedance spectroscopic (EIS) techniques (Sluyters, 1985) allows for the characterization of complex processes and interfaces by observation of a 
system's impedance response to a small periodic alternating perturbation at steady state (Dekker, 1969) or a voltage perturbation, a signal in the form below is applied:

$$
V(t)=V_{m} \sin (\omega t)
$$

where $V(t)$ is the overall signal with an amplitude $(V m)$ and an angular frequency $(\omega)$. The resulting current response is measured:

$$
i(t)=I_{m} \sin (\omega t+\vartheta)
$$

Please note that there is a phase difference, $\vartheta$, between the input and the output sinusoidal signals due to the capacitive nature (or inductive, or both) of the system. The impedance at a particular frequency is then calculated simply by the following relationship:

$$
Z(\omega)=V(t) / i(t)
$$

EIS is a very sensitive technique, low power and is complementary to many potential or current sweep techniques which usually operate away from the steady state. A broad frequency range $\left(10^{-4}-10^{6} \mathrm{~Hz}\right)$, the ability to average steady state responses, and simplification of current-potential behavior over a wide range of overpotential make electrochemical impedance approaches beneficial for analysis of transient phenomena such as diffusion and kinetics. Despite the advantages listed above, transformation of output data into meaningful physical/chemical interpretations is not straightforward, and physical and/or mathematical modeling is often needed as Impedance responses at all frequencies are first plotted against frequency spectrum in bode diagram like plots. A more common way of representation of data is to then plot the real and imaginary impedance responses versus each other on a Nyquist plot. Since this plot also contains the frequency information associated a particular impedance measurement, it is widely used in impedance analysis.

In this experiment, washing of the electrode with copious amounts of DI water, cyclic voltammetry was performed on the bare gold electrode using a three electrode system (Au working electrode, Pt counter electrode and $\mathrm{Ag} / \mathrm{AgCl}$ reference electrode) in a solution of $5 \mathrm{mM}\left[\mathrm{Fe}(\mathrm{CN})_{6}\right]^{3-} /\left[\mathrm{Fe}(\mathrm{CN})_{6}\right]^{4-}, 0.1 \mathrm{M} \mathrm{KCl}$ and $10 \mathrm{mM}$ PBS $(\mathrm{pH}=7.4)$ to obtain the formal potential and a peak-to-peak separation of $\sim 60 \mathrm{mV}(59 \mathrm{mV}$ theoretical). Impedance measurements were taken subsequently from the electrode at the former potential obtained from CV's at a frequency range of $0.1 \mathrm{~Hz}$ to $10^{5} \mathrm{~Hz}$, with amplitude of $5 \mathrm{mV}$. All electrochemical measurements were done with a $\mathrm{CHI660C}$ analyzer ( $\mathrm{CH}$ Instruments Inc.). The resulting data from this method is a plot of the real impedance versus the imaginary impedance coined a Nyquist curve (Fig. 5). From these Nyquist curves, several key parameters and system operations were then deduced. Namely, solution resistance, double layer capacitance (or constant phase elements formation) and electron transfer resistance were calculated to measure solution stability, surface properties or diffusion issues.

\subsection{Data Analysis and Optimal Frequency Analysis}

Data was placed into an EXCEL template after a concentration gradient was run. The spreadsheet analyzed the frequency versus impedance at each frequency at each concentration. Looking at the responses for each frequency across the concentrations, linear and log fit slopes and R-squares (degree of fit) were calculated and slope and R-square were plotted against frequency (Fig. 6 A). Determining 
ideal frequency is simply determining best R-square and slope and then plotting the impedance at that frequency against all concentrations and a resulting calibration curve is formed (Fig. 6 B).

In the case of IL-12, a lab model target protein, direct assay with anti-IL-12 immobilized onto he sensor surface yields a slope response (Fig. $7 \mathrm{~A}$ ) and R-square response (Fig. $7 \mathrm{~B}$ ) that result in a $5 \mathrm{~Hz}$ correlation curve (Fig. 8). The major hurtle seen in this approach is that many biomolecules of interests have frequency spectra that can often overlap or be very near one another (Fig. 9). Hence the concept of tuning was made. By application of a metal or core-shell particle, perhaps one could alter (or tune) the EIS frequency spectra of optimized binding.

\section{Method of Conjugation}

The method used to prepare the gold nanoparticles used in this study was described earlier in the literature (Slot, 1985). Nanoparticles of 2-20 nm in diameter were prepared according to this method.1 $\mathrm{mL}$ of $1 \%(\mathrm{w} / \mathrm{v}) \mathrm{HAuCl}_{4}$ was mixed with $79 \mathrm{~mL}$ of deionized (DI) water and heated to $60^{\circ} \mathrm{C}$ on a hot plate to yield gold nanoparticles of $5 \mathrm{~nm}$ size. Meanwhile, a reducing mixture of $4 \mathrm{~mL}$ of $1 \%(\mathrm{w} / \mathrm{v})$ trisodium citrate, $5 \mathrm{~mL}$ of $1 \%$ tannic acid and $5 \mathrm{~mL}$ of $2.5 \mathrm{mM} \mathrm{K}_{2} \mathrm{CO}_{3}$ and $5 \mathrm{~mL}$ DI water was brought to $60^{\circ} \mathrm{C}$ and added to the gold solution with stirring. The resulting solution is boiled for 10 more minutes after appearance of red color. Lower amounts of tannic acid and potassium carbonate were used in equal amounts to prepare larger particles (down to $0.01 \mathrm{~mL}$ for $20 \mathrm{~nm}$ particles) while keeping the total reducing solution volume at $20 \mathrm{~mL}$ and the final solution was cooled down to room temperature. Plasmon absorption at $520 \mathrm{~nm}$ was adjusted to $1 \mathrm{a}$.u. before use. If needed, a second system can be made in parallel and the two can be combined for co-immobilization (Fig. 10).

Lypholized antibodies and antigens were purchased from R\&D Systems, Minnesota, MN and were reconstituted in phosphate buffered saline (PBS). The latter was purchased in tablet form from CalBioChem, La Jolla, CA, and was used to prepare a working solution of $140 \mathrm{mM} \mathrm{NaCl}, 10 \mathrm{mM}$ phosphate buffer and $3 \mathrm{mM} \mathrm{KCl}, \mathrm{pH} 7.4$ at $25^{\circ} \mathrm{C}$. Minimum amount of antibody required to stabilize the gold nanoparticles was found by incubating $1 \mathrm{~mL}$ of gold solution with a series of protein solutions at varying concetnrations (10-100 ug/mL, $1 \mathrm{~mL}$ ) for $5 \mathrm{~min}$. After $5 \mathrm{~min}, 0.5 \mathrm{~mL}$ of $10 \%(\mathrm{w} / \mathrm{v}) \mathrm{NaCl}$ was added and the color of the solution was noted. The concentration, just above that of the solution in which the color turned blue from red, was deemed the minimum required amount for stabilization. Up to $25 \%$ excess protein was used above the minimum concentration level to ensure a higher degree of stabilization. Subsequently, gold nanoparticles were incubated for a total of $20 \mathrm{~min}$ at room temperature with appropriate amount of antibody after bringing the $\mathrm{pH}$ of the colloidal suspension to $\mathrm{pH}$ 8-9 with $\mathrm{K}_{2} \mathrm{CO}_{3}$, which is close to the isoelectronic point of the immunoglobulin $\mathrm{G}$ (IgG) molecules. This solution was then transferred into an appropriately-sized Beckman Quick-Seal tube and centrifuged in a Beckman-Coulter Optima L-100 XP Ultracentrifuge at various g's (50000 g for $20 \mathrm{~nm}, 70000 \mathrm{~g}$ for 10 $\mathrm{nm}, 90000 \mathrm{~g}$ for $5 \mathrm{~nm}$ and $100000 \mathrm{~g}$ for $2 \mathrm{~nm}$ ) at $4^{\circ} \mathrm{C}$ for $1 \mathrm{hr}$ to filter out unconjugated antibody from the complexes. Dark red colored pellets obtained from centrifugation were then reconstituted in $10 \mathrm{mM}$ phosphate buffered saline. Conjugates were consumed immediately, although there reports in the 
literature that suggest that antibody-gold complexes containing sodium azide $\left(\mathrm{NaN}_{3}\right)$ can be stable at $4^{\circ} \mathrm{C}$ for months (Kramarcy, 1991).

Although experimental aspects of preparing gold conjugates with various biomolecules are rather simple, mechanisms behind the process are not really understood. Ccoupling of biological macromolecules with gold nanoparticles depends on covalent and electronic interactions such as, binding of protein to gold atoms through thiol linkages, ionic interactions between the negatively charged gold surface and the positively charged terminals on the protein, and interactions originating from absorption of hydrophobic protein groups on gold. One of the most prominent theories that describes the behavior of gold colloids is DLVO theory (named after the scientists Derjaguin, Landau, Verwey and Overbeek), which evaluates the forces between adjacent gold nanoparticles in solution (Derjagiun, 1941; Verwey, 1948). According to DLVO theory, gold nanoparticles experience opposing forces due to overlapping double layers of adjacent particles. This force results in stabilization of particles, preventing aggregation. Van der Waals interactions due to dipole-dipole, dipole-induced dipole, and dispersion forces are other means through which gold nanoparticles can interact with other molecules in solution. In a typical gold colloid solution, these forces coexist and are in balance with each other. However, upon addition of positively charged groups (such as proteins), the negative charges on gold particles are shielded from each other, leading to a reduction in the magnitude of opposing forces. This in turn causes attractive forces to get stronger, and eventually a breach of the repulsive energy barrier takes place in solution, causing the two species to form a conjugate. In case of addition of negatively charged species to gold colloids (such as citrate groups employed in this study), repulsive interactions get stronger, further stabilizing the adjacent gold particles against aggregation. Although the mechanisms responsible for the attachment of biomolecules on AuNP's are multi-faceted, the detection thereof is straightforward by spectrophotometric methods.

The conjugation of gold nanoparticles with cytokine receptors, such as anti-IL-12, was verified by monitoring the surface plasmon resonance bands of colloidal gold. Due to localization of surface plasmon resonance oscillations on gold, the nanoparticles absorb in the UV-VIS region strongly at certain characteristic wavelengths. Absorption frequency primarily depends on particle size and dielectric medium surrounding the surface of the particles. For a given particle size, shifts in plasmon resonance bands indicate to changes in local refractive index. Adsorption of biomolecules on a particle reduces the oscillation frequency, inducing a redshift in plasmon maxima (Fig. 11). $10 \mathrm{~nm}$ gold nanoparticles were found to have an absorption maximum at $516 \mathrm{~nm}$ by UV-VIS spectroscopy. This was in accordance with the values previously reported in the literature for similarly prepared (citrate-capped) nanoparticles (Liu, 2007; Shakila, 2007; Swift, 2009). Upon conjugation with a cytokine receptor, anti-IL-12, the plasmon peak shifted to a higher wavelength, $526 \mathrm{~nm}$. This redshift was indicative of successful conjugation and was due to the change in resonance excitation frequency of surface plasmon polaritons on gold. This change in frequency is a function of the average interparticle distance, and the attachment of positively charged protein sites on negatively charged gold helps the particles shield their charge, resulting in a reduction of interparticle distances compared to negatively charged ligand such as citrate groups(Elghanian, 1997; Englebienne, 1998). Thus, gold nanoparticle-based sensing schemes that 
exploit changes in frequency upon binding have been reported by numerous studies in the literature (Leuvering, 1980, 1983; Mirkin, 1996).

\section{Method of EIS Verification of Tuning and ELISA Cross-Reactivity Testing 6.1 EIS Verification of Tuning}

Concentration gradients of the target, IL-12, were prepared and run against the sensors for verification. The data was then analyzed for untuned (unconjugated) and tuned (conjugated) AuNP-anti-IL-12 pairs. Slope and R-square plots against frequency were compared and changes in impedance were noted(Fig. 12). The untuned AuNP-anti-IL-12 pair in Fig. 12 A (a) was slightly higher in slope and more impedance could be seen in the higher frequency range where it is normally found, however, in the case of the tuned sensor in Fig. $12 \mathbf{A}$ (b), the response was shifted to lower frequency. Likewise, the R-square of the untuned AuNP-anti-IL-12 pair in Fig. 12 B (a) is slightly higher and more degree of fitness can be seen in the higher frequency range where it is normally found, however, in the case of the tuned sensor in Fig. 12 B (b), the degree of fitness is shifted to lower frequency as it was the case for the slopes.

Fig. 13 illustrates sensor responses to varying antigen concentrations $\left(0-10^{4} \mathrm{pg} / \mathrm{mL}\right)$. The control group, which consisted of immobilized anti-IL12 antibody only, gave a maximal detection frequency at $5 \mathrm{~Hz}$ (Fig.Fig. 13, black curve, squares), in-line with earlier reports in the literature (Sadik, 2002; Fairchild, 2009). The optimum signal at $5 \mathrm{~Hz}$ was diminished upon tuning the antibody response with $10 \mathrm{~nm}$ gold nanoparticles (Fig.Fig. 13, red curve, circles). It was observed that the slope of the concentration gradient also decreased significantly. However, the optimum detection frequency was shifted to $1 \mathrm{~Hz}$ as a result of tuning, as can be seen from the response of the conjugated antibody (Fig.Fig. 13, blue curve, triangles). A new slope that was close to the original slope at $5 \mathrm{~Hz}$ was also recovered at $1 \mathrm{~Hz}$. This is a significant outcome as it demonstrates that the optimal response of an antibody could be suppressed at

its native frequency by conjugation with gold nanoparticles subsequently detected at another frequency.

\subsection{ELISA Cross-reactivity Testing}

In addition to the observed changes in surface plasmon polariton excitation frequencies of gold, conjugation of nanoparticles with biomolecules also induces changes in the behavior of the adsorbate. Once immobilized, biomolecules adsorbed on gold particles are not free-standing as they are in solution and rather assume a certain conformation on the surface. This results in the protein adapting a more rigid structure, as opposed to that of a molecule that is able to change its orientation and conformation rather freely in solution. This raises the question of how the activity of an antibody is affected by conjugation with a gold particle.

Although protein-conjugated gold nanoparticles have been successfully employed as biological markers for decades, instances of total loss (Horrisberger, 1977) or partial loss of specific activity of a protein upon conjugation have also been reported (Kellenberger, 1987; Park, 1986). The study by Bauer et. al. stated that the antibody used in their study did not have the same dominant orientation on all the particles, meaning that the availability of suitable exposed binding sites varies even within the molecules 
of the same type (Bauer, 1975). Thus, the activity of conjugated proteins should be evaluated on a case by case basis. As such, the affinity of the conjugated cytokine receptors used in this study (anti-IL12 and anti-TNF- $\alpha$ ) were evaluated versus their bare counterparts by a technique that is widely accepted as the gold standard among biochemical detection methods: enzyme-linked immunosorbent assay (ELISA).

Controlled experiments against the standards were performed for verification of individual activities of IL-12 and anti-IL-12, as well as the method and conditions of immobilization of antibodies on ELISA plates. Factors such as the concentration of the primary antibody for coating the wells, the dilution factor for the horseradish peroxidase (HRP) and the concentration of the secondary -detection antibody were studied by a grid experiment where a series of primary antibody concentrations ( 1 $\mu \mathrm{g} / \mathrm{mL}, 5 \mu \mathrm{g} / \mathrm{mL}, 10 \mu \mathrm{g} / \mathrm{mL} 50 \mu \mathrm{g} / \mathrm{mL}$, and $100 \mu \mathrm{g} / \mathrm{mL}$ ), HRP dilution factors (1/200, 1/2000, and 1/2000) and secondary-antibody concentrations $(100 \mathrm{ng} / \mathrm{mL}, 200 \mathrm{ng} / \mathrm{mL}$, and $400 \mathrm{ng} / \mathrm{mL}$ ) were tested against each other to obtain the highest signal to noise ratio from the assay. The maximum signal to noise ratio and the dynamic range were found at primary antibody concentration of $10 \mu \mathrm{g} / \mathrm{mL}, \mathrm{HRP}$ dilution of $1 / 200$ and secondary antibody concentration of $100 \mathrm{ng} / \mathrm{mL}$ for IL-12.

Fig. 14 A illustrates the response of the bare antibody, anti-IL-12, towards its target, IL-12 from an average of three experiments under the conditions described above. A linear response was observed over the concentration range tested and the activity of the receptor-antigen couple was verified. A slope of $7.3 \times 10^{-5}$ and a linear regression constant $\left(R^{2}\right)$ of 0.99 was obtained from the response curve. The limit of detection (LOD) was determined by IUPAC method at a confidence level of $99.86 \%$ (Currie, 1999; Lee, 2003), and was found to be $3.9 \mathrm{pg} / \mathrm{mL}$ for this pair, which was much lower than the mean levels of IL-12 in plasma (114 pg/mL), indicating to the sensitivity of the assay (Castle, 2002). Activity of anti-IL-12 conjugates were evaluated similarly after optimization and verification of the assay. Antibody conjugates were immobilized on ELISA plates under the same conditions as their bare counterparts. Fig. The response of IL-12 towards anti-IL12 conjugates were illustrated in Fig. 14 B (black, squares). As can be seen from Fig. $14 \mathrm{~A}$, a highly linear response $\left(R^{2}=0.99\right)$ was obtained from the IL-12 conjugate assay (black, squares) within the concentration range $125-16000 \mathrm{pg} / \mathrm{mL}$ by using $20 \mathrm{~nm}$ particles. The slope of the curve was found to be $3.6 \times 10^{-5}$ and the limit of detection was $60 \mathrm{pg} / \mathrm{mL}$,

It is important to note the increase in LOD of the assay. Although this value is significantly higher than the limit of detection of the bare assay, it corresponds to approximately half of the plasma concentrations of IL-12 (Castle, 2002). This increase in LOD was driven by two major factors: loss of activity of the antibody upon conjugation and nonspecific binding on exposed gold surfaces. As mentioned earlier in the text above, the loss of activity of the antibody results from the changes in orientation and conformation of the conjugated molecules compared to their free counterparts in solution. However, it is evident from the LOD, slope and the dynamic range of the conjugate responses that a significant loss of activity was not the case in this system. Nonspecific binding of antigen on uncovered gold, on the other hand, depends primarily on the size of the probes employed. Due to geometrical considerations, the smaller the size of the particle, the lesser the probability that the protein will find sterically suitable sites on gold. The surface coverage, however, increases with particle size. One should also note that larger particle sizes have been associated with smaller probe densities in 
cell membranes (Harven, 1984) so a compromise between the size and immobilization efficiency must be found should gold probes are intended for immunohistochemistry applications. Since obtaining high target densities was not among the concerns of this study, larger gold particles $(20 \mathrm{~nm})$ were used to minimize the exposed surfaces and non-specific sites on gold to increase the limit of detection of the assay.

To test the extent of non-specific binding on IL-12 conjugates, another molecule from cytokine family, TNF- $\alpha$ was used. Fig. 14 B shows cross responses of anti-IL-12 conjugates towards TNF- $\alpha$. As it is evident from Fig. 14 B (red, circles), cross reactivity of TNF- $\alpha$ was indistinguishable from the blank response, indicating to very low level of non-specific binding under these conditions. Ethanolamine, a blocking agent used in this study, further contributes to decreasing nonspecific binding due to its small size and efficiency of masking the exposed gold surfaces against other molecules in solution. This may play an important role in decreasing non-specific binding in samples including more than one conjugate immobilized on the same sensor for simultaneous frequency-based multiplexed detection. Similar results were obtained from an assay containing the conjugated/unconjugated forms of another important biomarker, TNF- $\alpha$ as discussed below.

Fig. 15 A depicts the response of bare TNF- $\alpha$ assay. As was the case with IL-12, the conditions of the assay were optimized beforehand with a grid experiment. Optimum primary antibody concentration, HRP dilution factor, and the secondary antibody concentration were found to be $10 \mu \mathrm{g} / \mathrm{mL}, 1 / 200,200$ $\mathrm{ng} / \mathrm{mL}$, respectively. The response was highly linear $\left(\mathrm{R}^{2}=0.99\right)$ within the concentration range tested with a slope of $4.3 \times 10^{-4}$ and a limit of detection similar to that of IL-12, at $3.4 \mathrm{pg} / \mathrm{mL}$. Upon verification of TNF- $\alpha$ activity, anti-TNF- $\alpha$ antibodies were conjugated with $20 \mathrm{~nm}$ gold nanoparticles for evaluating theie activity and cross-reactivity with other molecules.

Fig. 15 B shows the results obtained from ELISA assays of TNF- $\alpha$ conjugates. It can ben seen from the Fig. that TNF- $\alpha$ conjugates were able to recognize their own antigens over a wide range of concentrations (black, squares). The linearity was high $\left(R^{2}=0.99\right)$, and the slope was $4.3 \times 10^{-5}$. The limit of detection was found to be $109 \mathrm{pg} / \mathrm{mL}$, which was higher than the limit of detection for the bare TNF- $\alpha$ assay. These findings suggest that the affinity of anti-TNF- $\alpha$ towards the target cytokine was retained to a great degree after conjugation. The loss in activity was possibly due to factors mentioned earlier in the text, such as the change in orientation and conformation of the immobilized TNF- $\alpha$ on gold and possibility of having exposed gold sites that increased non-specific binding. The cross reactivity of TNF- $\alpha$ conjugates were also evaluated. As shown in Fig. 15 B (red, circles), AuNP-TNF- $\alpha$ complexes did not interact with IL12 to an appreciable degree over a wide concentration range, which can be attributed to efficient blocking of nonspecific binding sites on gold. However, it was noted that the target responses, particularly at lower concentrations, were higher than the blank signal, indicating to limited yet quantifiable responses from conjugated TNF- $\alpha$ complexes towards IL-12 molecules. Please note that this effect was much less pronounced in the case of IL-12 conjugates, pointing to unique differences in activities of the two conjugated antibodies as a result of their molecular structures on gold. 


\section{Troubleshooting}

\subsection{PCB fabrication and electroplating}

\subsubsection{PCB Fabrication}

Some issues can arise while making a printed circuit board, some are: underdeveloped PCB's due to mark not being dark enough; developing times get longer as you process more than one board; scratching of the resulting $\mathrm{PCB}^{\prime}$ s during cutting and separation; and the copper becoming dirty before electroplating. If the mask is not dark enough two means of producing a darker image are: print on two transparencies and tape together or using a fresh toner and printing a higher quality image. If reusing the bath to remove the photoresist or copper, note that the strength (and hence speed) of reaction will decrease after each use and by the fifth or sixth 6 " $\times 6^{\prime \prime}$ board, the baths will lose most chemical reactivity and should be replaced. To avoid scratching of the copper, and hence possible creating an open circuit on the PCB sensors, leave the photoresist on during separation or cutting. Also, leave on until ready to electroplate as the photoresist keeps the copper fresh and clean.

\subsubsection{Electroplating}

Electroplating is considered a very complicated process with over 29 factors that can result in a "bad" plate job (Dini, 1998). Some of the most common processing problems are easily solved by due diligence, such as $\mathrm{pH}$ and temperature issues; starting with a very clean copper surface; and of course, reuse of materials again can be a concern. Check temperature and $\mathrm{pH}$ as well as fill volumes before and during the run to maintain a constant temperature, $\mathrm{pH}$ and concentration in the bath solutions. After rinse with acetone and degreasing, immediately place array into nickel bath to prevent organics and other contaminants from ruining the clean copper surface. If reusing degreaser, heat on a hot plate with a cover to $93.3^{\circ} \mathrm{C}$. (This step takes a while, allow for at least an hour and set the hot plate to $235^{\circ} \mathrm{C}$ and continuously monitor temperature, make sure it gets no higher than $93.3^{\circ} \mathrm{C}$. If reusing gold and nickel solution (that are already in the water bath), check the levels and replenish with DI.

\subsection{Immobilization}

\subsubsection{Polishing}

Because the cleanliness of the electrode surface is critically important in ensuring proper biosensor functionality, a variety of techniques can be employed in order to minimize any potential problems. Electrochemical measurements such as CV and EIS should be run both before and after electrode polishing in order to determine whether successive immobilization steps can be completed. This includes measuring the oxidation and reduction potentials from the CV measurements in order to calculate a formal potential for each electrode so that the bare electrodes can be run using EIS. If the surface resistance is negligible $(<30 \mathrm{Ohms})$, the surface of the electrode is thought to be cleaned sufficiently for the immobilization process. This number is flexible depending on the type of electrode and molecular recognition element being employed, and serves as a guide from our group's prior work with gold disk electrodes. Obviously, minimizing the surface resistance of the electrodes is ideal before beginning any type of immobilization process. If the surface resistance remains quite large after polishing, it is recommended that electrodes be repolished in order to ensure a clean surface. Because of the numerous steps, time, and reagents involved, having the cleanest electrode surface possible to start with will minimize potential immobilization problems later in the process. A variety of methods 
can be used for polishing gold disk electrodes. Our group has found success with first polishing the electrodes by hand using alumina powder and microfiber clothes, followed by sonication to remove excess debris using acetone and water. The sizes of the alumina powder used and sonication times are at the investigators discretion depending on the particular biosensor application.

\subsubsection{Rinsing and Storage}

A common problem encountered during the immobilization process occurs when rinsing the electrodes with PBS after incubation with the alkanethiol, EDC/NHS, and ethanolamine solutions. Distilled water and PBS are common rinsing reagents used in immobilization and, while inert, care must be taken in applying these solutions appropriately. Rinsing should be completed in a way that maintains the integrity of the electrode surface to the greatest degree. As such, the electrode surface should not be rinsed directly but instead should be gently rinsed at an angle in order to minimize shear forces that could potentially unconjugate the immobilized linkers and molecular recognition elements on the electrode surface. Additionally, the gold disk electrodes should be gently hand dried to remove any excess PBS or distilled water from the electrode surface. This will help to ensure that next reagent used in the immobilization process is not inadvertently diluted or prevented from contacting the sensor surface. Each of the gold disk electrodes should be stored dry after incubation, and a method should be employed to prevent outside air from contacting the sensor surface as much as possible. A method that our group found helpful was to construct wells that the electrodes could be placed in and covered with parafilm. For long term storage ( $>1$ day), the gold disk electrodes should be refrigerated dry at $4^{\circ}$ C until use.

\subsection{Formal Potential and Optimal frequency determination}

The EIS technique perturbs an electrochemical system, in this case redox probe, with an AC voltage about a DC voltage offset supposedly corresponding to the equilibrium point of the redox probe components, or the formal potential. The equilibrium point is also the point at which the system will respond most strongly (current-wise) to the AC signal. If all sensors in an EIS experiment share a common reference electrode, then determination of a correct DC voltage offset is of less importance as the data from all the electrodes will at least be affected in the same manner by this neglect. The PCB electrodes have individual references, and as such require more attention.

In a 3-Electrode electrochemical cell, reference electrodes are relied on to maintain a constant voltage offset from the working electrode in any given solution. Sensors with individualized reference electrodes, like the $\mathrm{Ag} / \mathrm{AgCl}$ references plated onto the PCB sensor chips, will each have a slightly different offset between the working and reference electrodes, and will consequently have different formal potentials. Averaging the peaks of a Cyclic Voltammetry plot will obtain the formal potential of a 3-electrode system. Thus CV can be used to obtain the individual formal potentials for each PCB sensor chip in redox probe. Without using individualized formal potentials, EIS experiments using PCBs will each be perturbing the solution a different distance from its equilibrium, hence each would have less comparable outputs (i.e. less useful data). By using these individualized formal potentials (measured by CV) as the DC offset for EIS experiments, this problem is bypassed, as well as the signal from the PCB sensors increased. 
Data of frequency versus impedance at different target concentrations were interpreted to develop the frequency of maximum response (henceforth optimal frequency), that is, the frequency in the $A C$ sweep at which impedance was most responsive to changing concentrations of a particular antigenantibody conjugation. In order to determine the optimal frequency, a plot was made of frequency versus percent change in impedance over all the cytokine concentrations tested. This was also compared to a plot of frequency versus the coefficient of determination as determined from linear fits of impedance versus concentration at every employed frequency. Thus, an optimal frequency was found where impedance was very responsive to concentration change in a manner conforming to the log of concentration. This optimal frequency was identified for specific antibody-antigen binding events.

\subsection{Conjugation}

Protocols used for conjugation of gold nanoparticles to antibodies in this study rely on covalent and electrostatic attractions between constituents. This area is rather well-studied in the literature but the mechanisms behind the process are poorly understood. Therefore special care needs to be taken throughout to ensure the quality of the end-product. Preparation of gold nanoparticles is a straightforward protocol during which colloidal particles are formed from $\mathrm{HAuCl}_{4}$ by means of a reducing solution, which also includes the ligand that stabilizes the newly-formed particles (Slot, 1985). Resulting mixture needs to be cooled on ice and the $\mathrm{pH}$ should be adjusted to 8.5 with $0.5 \mathrm{M}$ $\mathrm{NaOH}$. During this adjustment, $\mathrm{pH}$ paper should be used rather than $\mathrm{pH}$ electrodes as unstable gold nanoparticles can be damaging to electrode membranes. The next step is the titration of antibody-gold solutions for determination of optimal concentrations. Since most commercial antibodies include stabilizing agents such as BSA or gelatin, care must be taken to minimize the binding between these agents and gold nanoparticles.

Antibodies can be affinity-purified before conjugation to maximize the interactions between the desired antibody and gold nanoparticles. Once titration is complete, lowest amount of antibody that is enough to stabilize the gold should be used for centrifugation. Solutions should be centrifuged for $1 \mathrm{hr}$ at $4^{\circ} \mathrm{C}$ at $120000 \mathrm{~g}$ for $2-3 \mathrm{~nm}$ gold and $100000 \mathrm{~g}$ for $5 \mathrm{~nm}$ gold. Excess centrifugation will result in increased aggregation and thus should be avoided. Prompt collection of conjugated concentrates is also crucial to avoid resuspension of the aggregates that are expected to form in the bottom portion of the sample. If desired, size distribution of the conjugates can be improved performing an additional centrifugation step at $200000 \mathrm{~g}$ for 30 mins at $4^{\circ} \mathrm{C}$ by means of a $10-40 \%$ glycerol gradient in PBS and $0.1 \%$ BSA. The fractions from the lower half should be avoided (collect top 20 fractions) as they will contain aggregates. Characterization of the resulting conjugates by UV-VIS spectroscopy and subsequent EIS measurements should be done promptly to ensure freshness of the samples used. However, as noted earlier, sodium azide $\left(\mathrm{NaN}_{3}\right)$ has been employed in the literature to preserve the stability of the antibody-gold complexes for storage at $4^{\circ} \mathrm{C}$ for months (Kramarcy, 1991). 


\subsection{Verification by ELISA and EIS}

Enzyme-linked immunosorbent assay (ELISA) is an exceptionally well-studied method to study cytokines and thus was employed as the reference method to verify the activity of the conjugated proteins on gold nanoparticles. This technique is generally robust in many aspects however some pitfalls do exist that can result in no or reduced signal from the conjugates. It is safe to say that the most important factor in obtaining successful outcomes from ELISA's is the starting solutions. Due to the number and nature of the solutions that one needs during an ELISA, all solutions must be freshly prepared beforehand and consumed promptly without interruption. One of the pitfalls is washing between various steps such as blocking and immobilization which can lead to increased background noise when performed insufficiently. Wash buffer needs to be forcefully employed, however, should be directed away from the base of the cells to avoid stripping moderately stable material off the surface. Subsequent aspiration of the wash liquid should be as thorough as possible to minimize background and maximize the performance of the assay. Concentration ranges also must be carefully selected to ensure signal strength.

Similar caution should be exercised during electrochemical impedance measurements. All solutions, including antibody conjugates and 1-ethyl-3-(3-dimethylaminopropyl) carbodiimide (EDC), should especially be used shortly upon preparation and care must be taken to protect the latter from moisture heat (store at $-20^{\circ} \mathrm{C}$ ). Depending on the EIS electrode used, leakage from the wells or improper capping during incubation are also other areas of concern. The electrode carrying the conjugated antibodies should also be treated carefully as the detachment of the conjugates can be an issue if proper handling/pipetting techniques are not employed during washing/blocking.

\subsection{Discussion and Conclusion}

In this work, we have developed and described the techniques and methods to: 1) photofabricate electrochemical sensor platforms, 2) electroplate useful electrochemical metals, such as gold onto the PCB's, 3) covalently attach molecular recognition elements onto gold sensors, 4) tested sensors against targets in solutions, 5) created a novel method of screening EIS frequency for pertinent frequencies of optimal binding, 6) tuned adjacent frequencies away from one another, and 7) demonstrate that functionality is not lost in these systems due to the tuning or attachment. Of course, this work has been accomplished with much testing, optimizing concentrations, times, components, among other verification studies not herein. Simple demonstrative studies such as changing concentration of molecular recognition elements having little effect on impedance and frequency or verification of molecular recognition attachment surface concentration were not shown.

The implications of this work are far reaching. High throughput instrumentation could be developed to screen vast quantities of samples for choice markers. Another embodiment includes looking at single proteins for post translational modification variety in regards to disease diagnosis. One could also test for a variety of infectious diseases or pathogens using a simplified single senor in a critical care area. 
Simple point of care technologies, that function very similar to self-monitoring blood glucose meters could use multimarkers to better detect, diagnosis or monitor diseases at home by the patient.

The future directions of our work include realization of those technologies. To accomplish those lofty goals, there is much work to be done. In order to validate that multiple markers, at different frequencies can be monitored simultaneously, a new instrument that has the ability to generate and demultiplex a mixed sinusoidal or multisine signal must be developed. This could include a new potentiostat design or simple add-on hardware or software development. Sample related issues could also arise such as developing a sensor monitor for two markers, one found naturally at $\mathrm{pg} / \mathrm{mL}$ levels and the other in $\mathrm{mg} / \mathrm{mL}$. Would simply modifying the amount of antibody or molecular recognition elements suffice, or would alteration of the AC component of the EIS signal help (or hinder) detection. This and much more model development of the tuning are required before these devices could see application in bettering disease management and control. This work, obviously, is just the beginning.

\section{Acknowledgements}

This work was funding in part by BioDesign Institute at Arizona State University and, the Fulton School of engineering's Fulton Undergraduate Research Initiative (FURI) program.

\section{References}

Ala-Korpela, M, Kangas, AJ, Soininen, P. Genome Medicine 2012, 4:36, 1-4.

Bard, A. J.; Faulkner, L. R. Electrochemical Methods: Fundamentals and Applications; John Wiley \& Sons Inc.: New York, 2000.

Bauer, H.; Gerber, H.; Horrisberger, M. Experientia 1975, 31, 1149.

Bensi, G, Mora, M, Tuscano, G, Biagini, M, Chiarot, E, Bombaci, M, Capo, S, Falugi, F, Manetti, AGO, Donato, P, Swennen, E, Gallotta, M, Pinto, V, Chiappini, N, Musser, JM, Janilczyk, R, Mariani, M, Scarselli, M, telford, JL, Grifantini, R, Norais, N, Margarit, I, Grandi, G. Mole. Cell. Proteo. 2012 First Published on January 27, 2012, doi: 10.1074/mcp.M111.015693

Castle, P. E.; Hildesheim, A.; Bowman, F. P.; Strickler, H. D.; Walker, J. L.; Pustilnik, T.; Edwards, R. P.;

Crowley-Nowick, P. A. Journal of Clinical Immunology 2002, 22, 23.

Choi, J-W, Kang, D-K, Park, H, deMello, AJ, Chang, S-I. Analy Chem 2012. 84, 3849-54.

Currie, L. Anal. Chim. Acta 1999, 391, 127.

Davydov, D. N.; Sattari, P. A.; AlMawlawi, D.; Osika, A.; Haslett, T. L.; Moskovits, M. J. Appl. Phys. 1999, 96, 3983.

Dekker, B. G.; Sluyters-Rehbach, M.; Sluyters, J. H. J. Electroanal. Chem. 1969, 23, 9, 17, 137.

Derjaguin, B.; Landau, L. Acta Physico Chemica URSS 1941, 14, 633.

Dini, J.W. Electrodeposition Noyws Pub. Park Ridge USA, 1993.

Elghanian, R.; Storhoff, J. J.; Mucic, R. C.; Letsinger, R. L.; Mirkin, C. A. Science 1997, 277, 1078.

Englebienne, P. Analyst 1998, 123, 1599.

Fairchild, A. B.; McAferty, K.; Demirok, U. K.; La Belle, J. T. In IEEE/ICME 2009, p 1-5.

Ferain, E.; Legras, R. Nucl. Instrum. Methods B 1993, 84, 331.

Ferre, R.; Ounadjela, K.; George, J. M.; Piraux, L.; Dubois, S. Phys. Rev. B 1997, 56, 14066.

Haddon, R. C. Acc. Chem. Res. 2002, 35, 997.

Harven, E. D.; Leung, R.; Christensen, H. J. Cell Biology 1984, 99, 53.

Horrisberger, M.; Rosset, J. J. Histochem. Cytochem. 1977, 25, 295. 
Iwasaki, T.; Motoi, T.; Den, T. Appl. Phys. Lett. 1999, 75, 2044.

Kellenberger, E.; Durrenberger, M.; Villiger, W.; Carlemalm, E.; Wurtz, M. J. Histochem. Cytochem. 1987, 35, 959.

Kramarcy, N. R.; Sealock, R. J. Histochem. Cytochem. 1991, 39, 37.

La Belle, JT, Gerlach, JQ, Svarovsky, S, Joshi, L. Analy Chem. 2007, 79, 6959-64.

La Belle, JT, Demirok, UK, Patel, DR, Cook, CB. Analyst. 2011, 136, 7, 1496-1501.

Lee, P. W.; Aizawa, H.; Barefoot, A. C.; Murphy, J. J. Residue Analytical Methods for Agrochemicals; John

Wiley \& Sons, 2003.

Leuvering, J. H. W.; Goverde, B. C.; Thal, P. J. H. M.; Schuurs, A. H. W. M. Immunol. Methods 1983, 60, 9. Leuvering, J. H. W.; Thal, P. J. H. M.; van der Waart, M.; Schuurs, A. H. W. M. Immunoassay 1980, 1, 77.

Li, D, Luo, J, Han, L, Peng, Y, Zhong, D. Adv Matl Res Vols. 2012. 396-8, 1914-7.

Li, Z. M.; Tang, Z. K.; Liu, H.; Wang, N.; Chan, C.; Saito, R.; Okada, S.; Li, G. D.; Chen, J. S.; Nagasawa, N.; Liu, P, Gu, X, Zhao, X, Liu, X. Adv Matl Res Vols. 2012. 341-2, 411-5.

Liu, Y.; Shipton, M. K.; Ryan, J.; Kaufman, E. D.; Franzen, S.; Feldheim, D. L. Anal. Chem. 2007, 79, 2221.

McNaught, A. D., Wilkinson, A. "IUPAC Compendium of Chemical Terminology." Blackwell Scientific Publications. 2nd Edition (1997).

Mirkin, C. A.; Letsinger, R. L.; Mucic, R. C.; Storhoff, J. Nature 1996, 382, 607.

Mu, S, Wang, X, Li, Y-T, Li, D-W, Long Y-T. Analyst 2012. 137, 3220-3.

Park, K.; Albrecht, R.; Simmons, S.; Cooper, S. J. Colloid Interface Sci. 1986, 111, 197.

Rabinovich, R. IEEE Comm 2012. 50:4, 129-33.

Sadik, O.; Xu, A. H. Anal. Chem.. 2002, 74, 3142.

Saedi, M. R.; Ghorbani, M. J. Mater. Chem. 2005, 91, 417.

Shakila, V.; Pandian, K. J. Solid State Electrochem. 2007, 11, 296.

Sloan, J.; Kirkland, A. I.; Hutchison, J. L.; Green, M. L. H. Acc. Chem. Res. 2002, 35, 1054.

Slot, J. W.; Geuze, H. J. Eur. J. Cell Biol. 1985, 38, 87.

Stanley, CE, Wootton, RCR, deMello, AJ. CHIMIA Internat/ J Chem, 2012, 66: 3, 88-98.

Sluyters-Rehbach, M.; Sluyters, J. H. J. Electroanal. Chem. 1969, 23, 457.

Swift, J.; Butts, A.; Cheung-Lau, J.; Yerubandi, V.; Mochowski, I. J. Langmuir 2009, 25, 5219.

Tsirigos, A, Haiminen, N, Bilal, E, Utro, F. Bioinformatics 2011. 28:2 282-3.

Tsuda, S. Phys. Rev. Lett. 2001, 87, 127401.

Verwey, E. J. W.; Overbeek, J. T. G. Theory of the stability of lyophopic colloids; Elsevier: Amsterdam, 1948.

Wang, N.; Tang, Y. H.; Zhang, Y. F.; Lee, C. S.; Lee, S. T. Phys. Rev. B 1998, 58 , R16024.

Weston, AD, Hood, L. J Proteome Res. 2004. 3:2, 179-96.

Workman, P, Clarke, PA, Al-Lazikani, B. Cancer Cell 2012, 21:4, 455-8.

Zeng, H.; Zheng, M.; Skomski, R.; Sellmyer, D. J.; Liu, Y.; Menon, L.; Bandyopadhyay, S. J. Appl. Phys. $2000,87,4718$. 
A

B

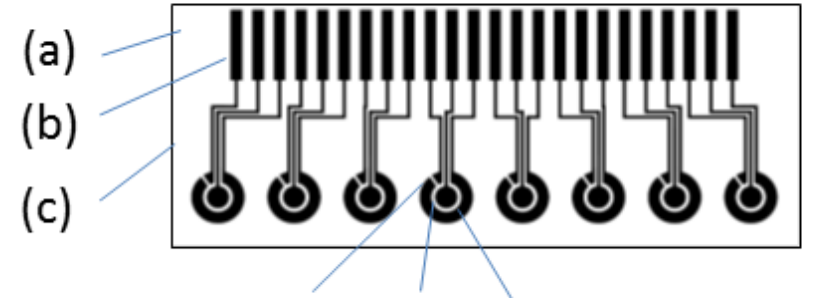

(d) (e) (f) (a)

(b)

(c)

(d)

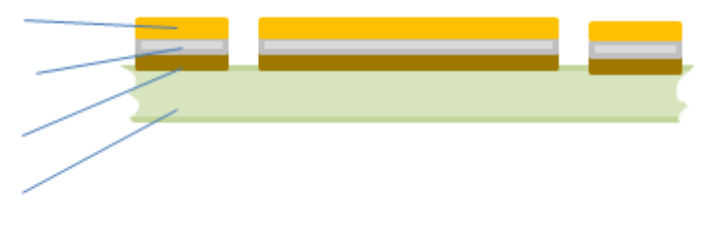

Fig. 1 - In A the typical printed Circuit Board electroplated 3 electrode lead electrochemical sensor array with 8 sensors showing (a) PCB substrate, (b) electrical leads for connection, (c) leads running to sensors, (d) small reference electrode, (e) center working electrode and (f) circulating counter electrode/ In B the side view schematic showing the various layers of the PCB (a) substrate, (b) copper cladding, (c) nickel plating, and (d) gold plating.
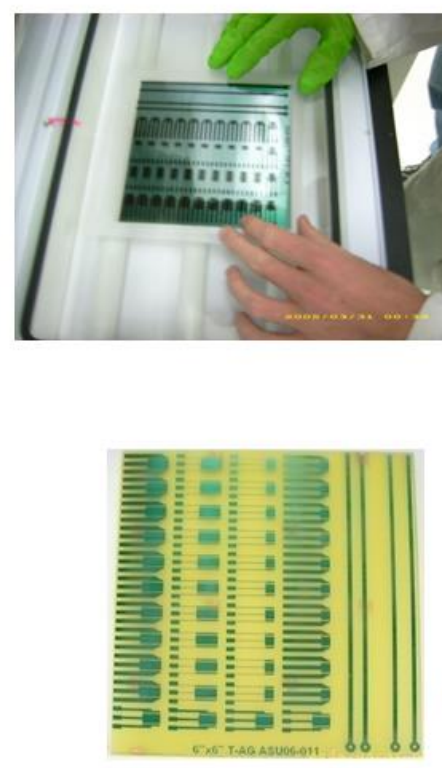

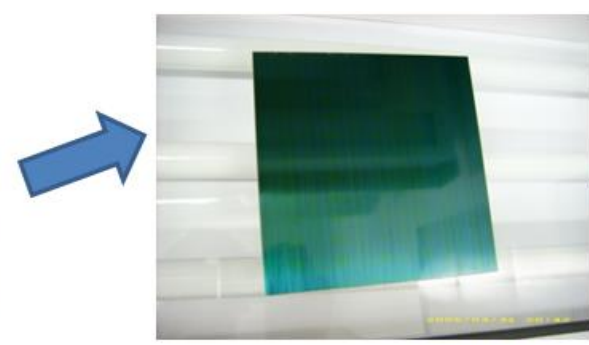

(b)

(a)

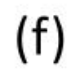

(f)

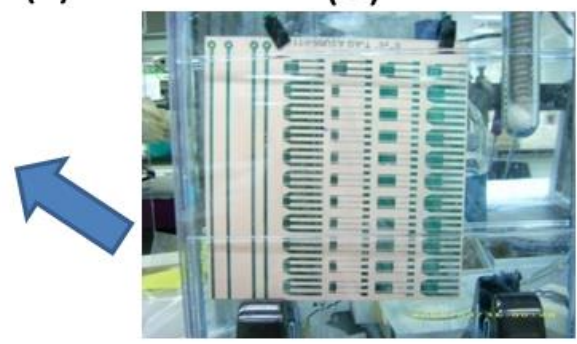

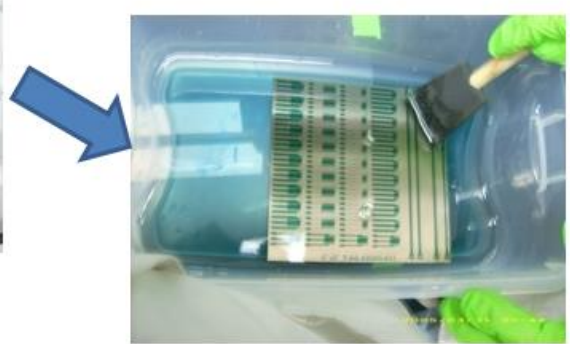

(c)

(d)

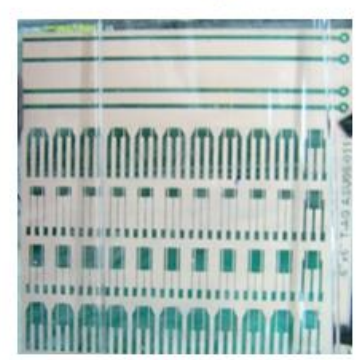

Fig. 2 - The first step of the photofabrication process (a) a mask or template is made using computer aided design software and printed onto a transparency and placed on top of a pre-sensitized copper clad board in a UV light box, (b) the board is exposed for a pre-determined amount of time, (c) the exposed photoresist is removed in a bath of $\mathrm{pH} 14.0$ sodium hydroxide developer for $2 \mathrm{~min}$, (d) the excess developer is rinsed off using distilled water stopping the chemical reaction, (e) the board is now 
placed into a pH 1.0 solution of ammonium persulfate to remove the unprotected copper, thus leaving (f) a series of sensors or arrays ready for electroplating.

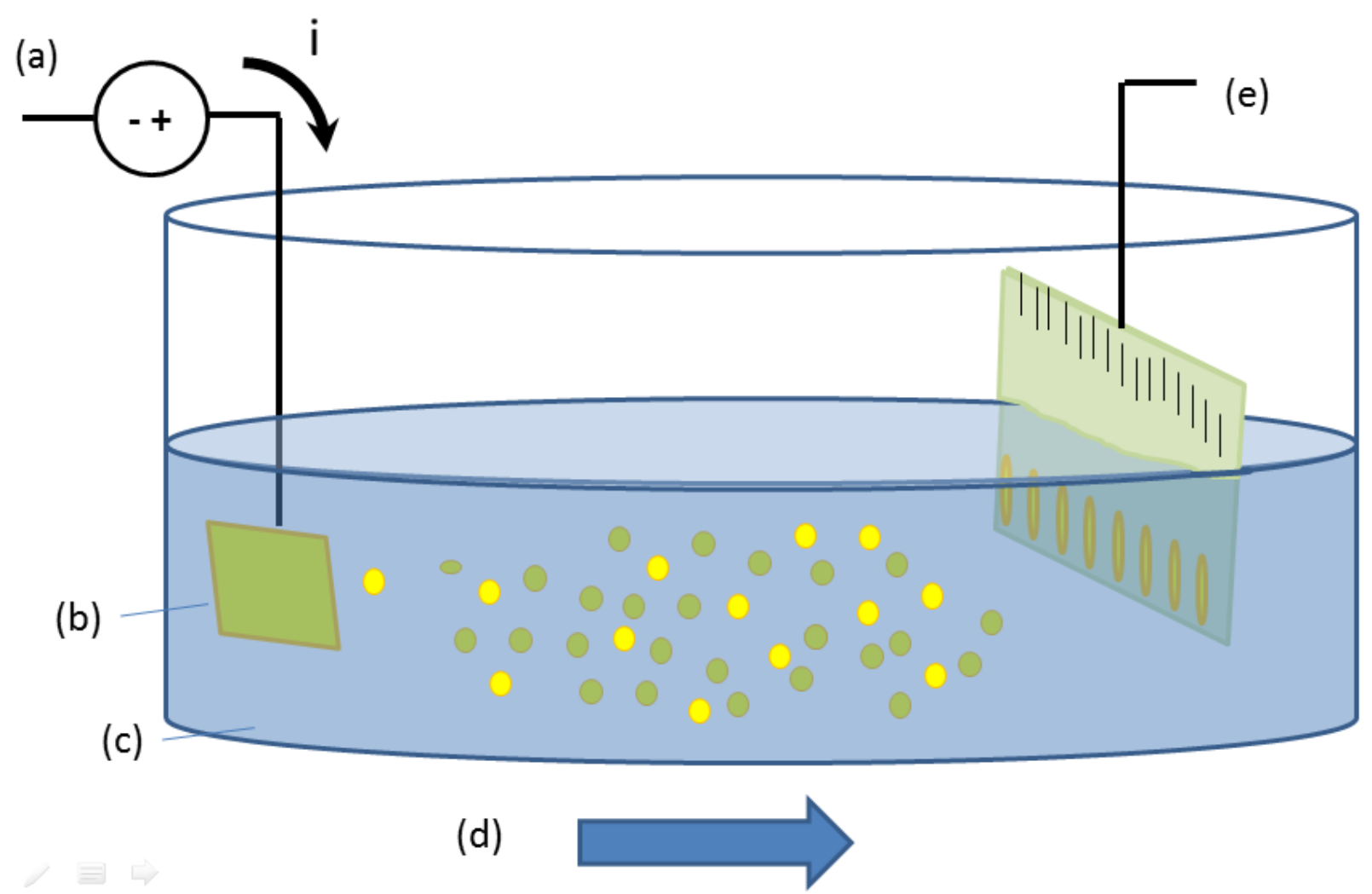

Fig. 3 - Schematic of electroplating process whereby a current induced stripping of (b) host material (e.g. nickel or gold) in a (c) salt solution of same material induces a (d) flow of the material to be plated on the (e) electrode array material acting as the other half of the electrical circuit. 
(c)

(b)

(a)

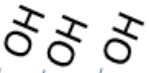

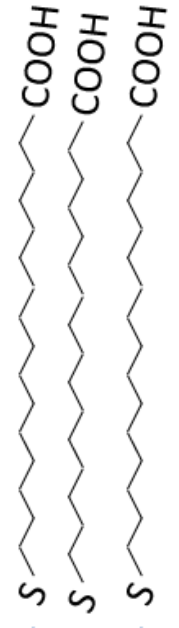

(d)

(e) (d)

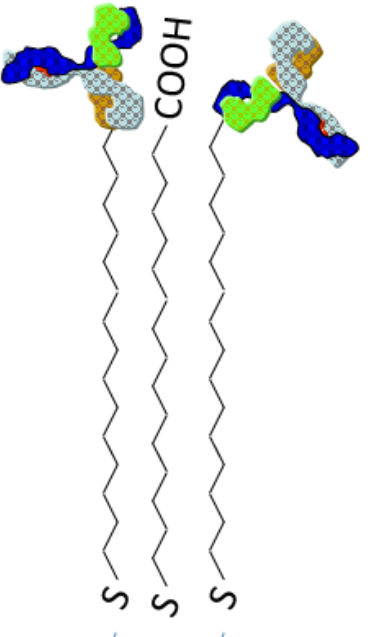

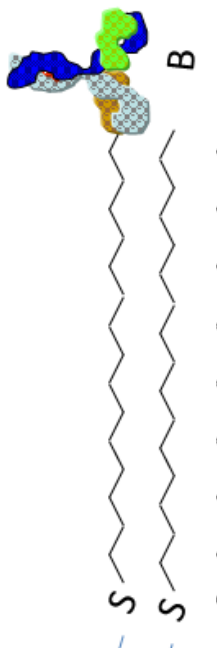

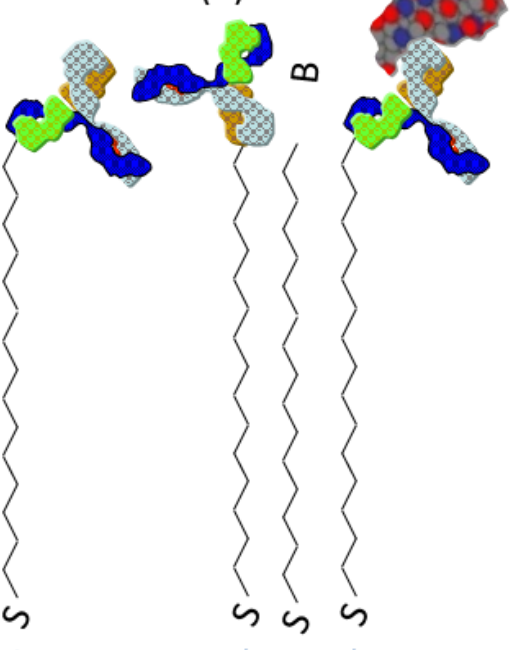

Fig. 4 - (a) Surface preparation is typically made possible by polishing a gold surface or electroplating/sputtering/etc a fresh gold surface, leaving hydroxyls groups on the clean surface where self-assembled monolayers can be made with a (b) a linker molecule such as 16-mercaptohexadeconic acid (16-MHDA) Monolayer, which in turn can be (c) activated using zero crosslength linkers such as (EDC/NHS), rapidly accepting an (c) antibody immobilization, with free 16-MHDA terminal end groups being block by the (d) blocking molecule ethanolamine and detection can occur under (e) target binding.

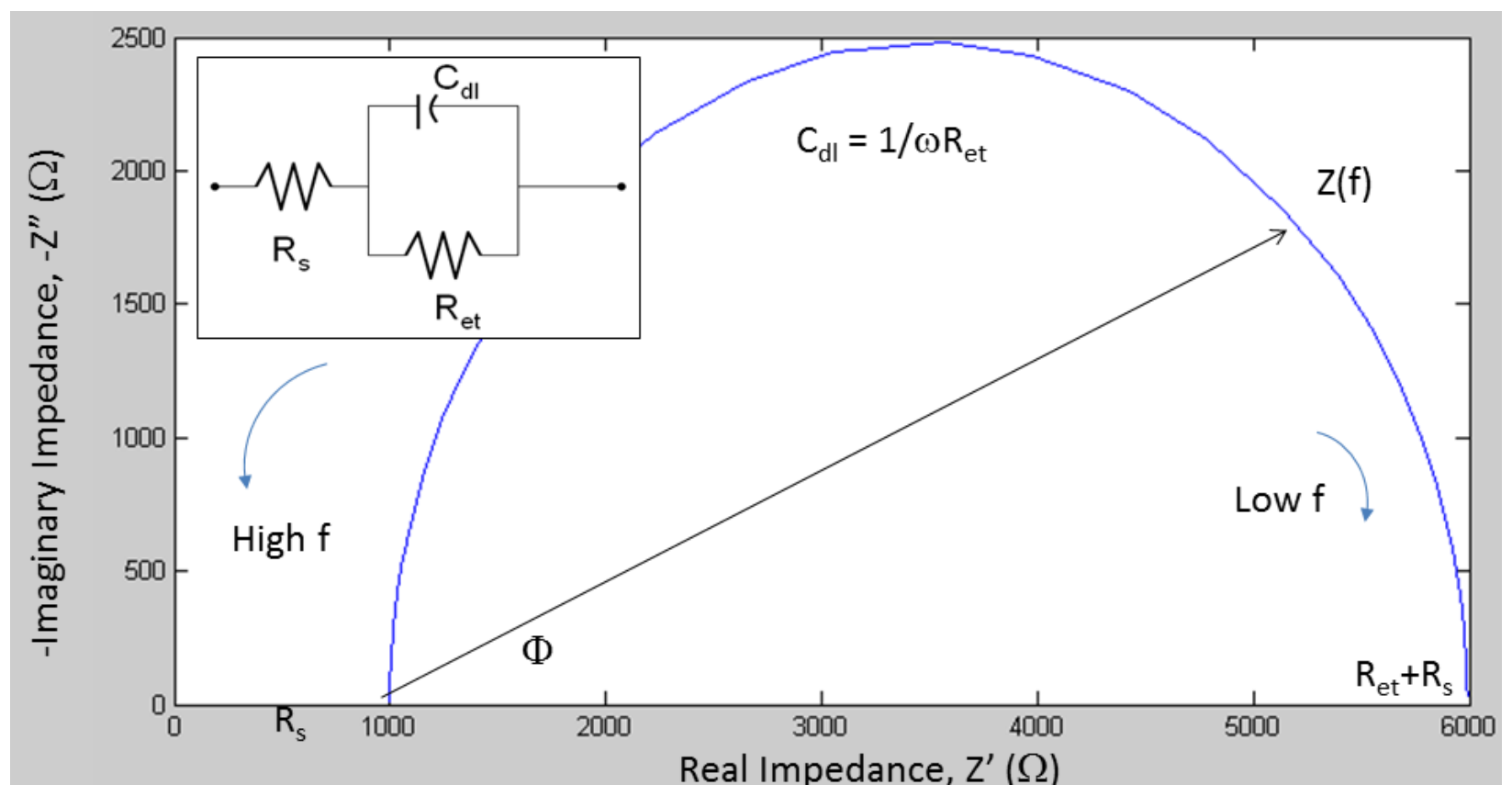


Fig. 5 - Impedance spectra, Nyquist curve, a plot of the real impedance versus imaginary impedance. The graph is semicircular with higher frequencies associated near the origin and lower ones away from the origin. Key circuit parameters are given as well as (INSET) equivalent circuit diagram.

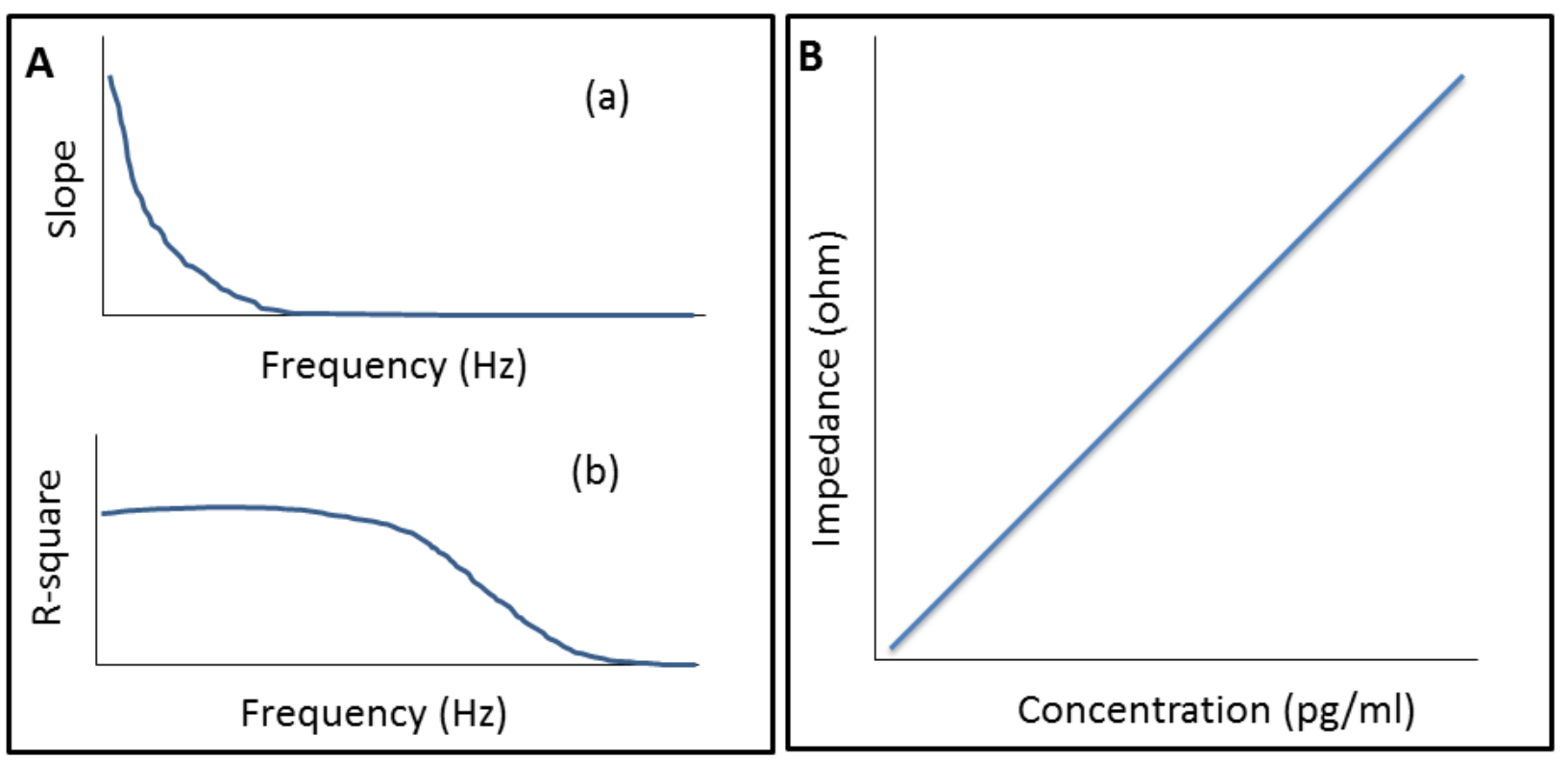

Fig. 6 - Schematic representation A: diagrams for (a) slope and (b) R-square versus frequency which are used to isolate B: optimized impedance concentration curves.

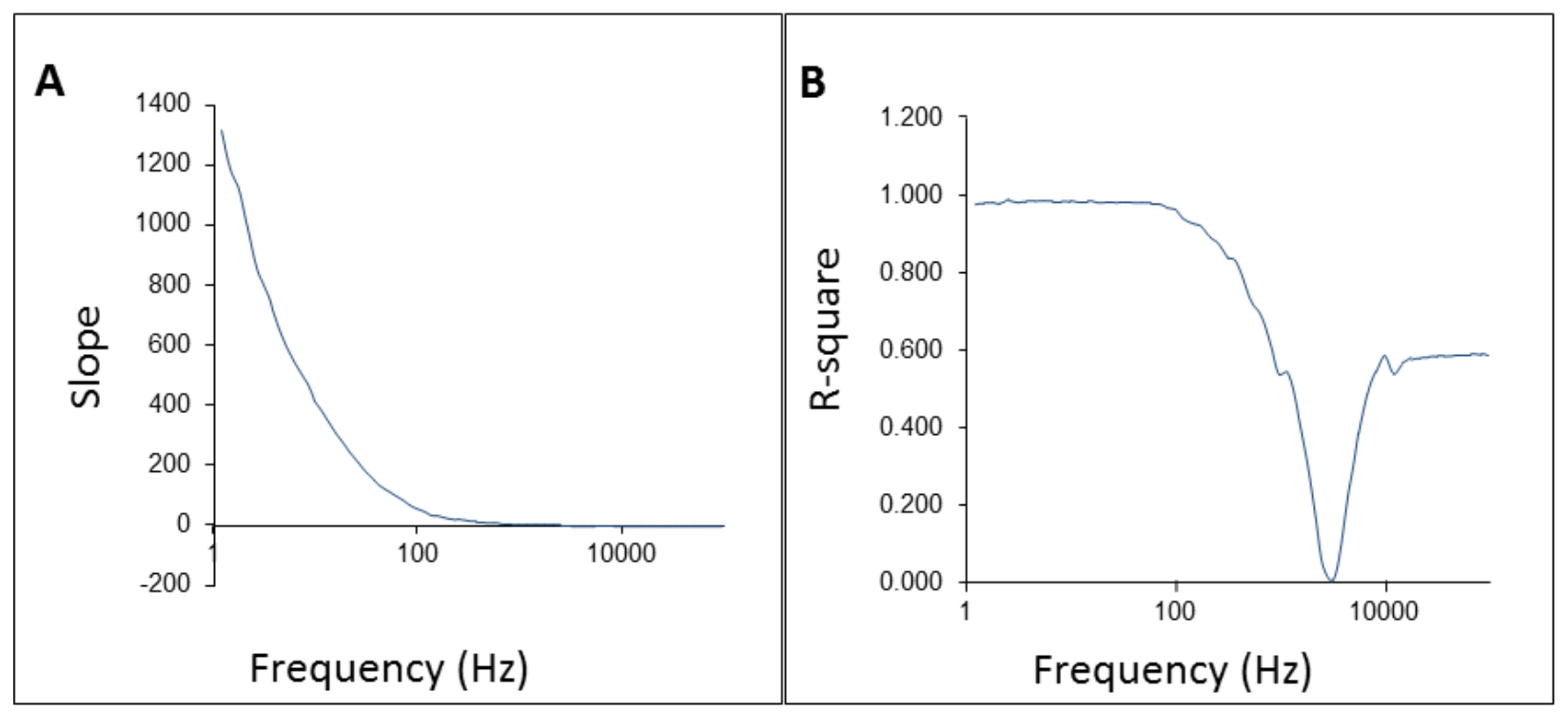

Fig. 7 - A concentration gradient of interleukin-12 (IL-12) made against anti-IL-12 sensor with A: the slope versus frequency plot showing high degree of responsivity at low frequencies but coupled to this is B: the R-square or degree of fitness versus frequency plot showing optimal binding is associated with a certain range of frequencies 


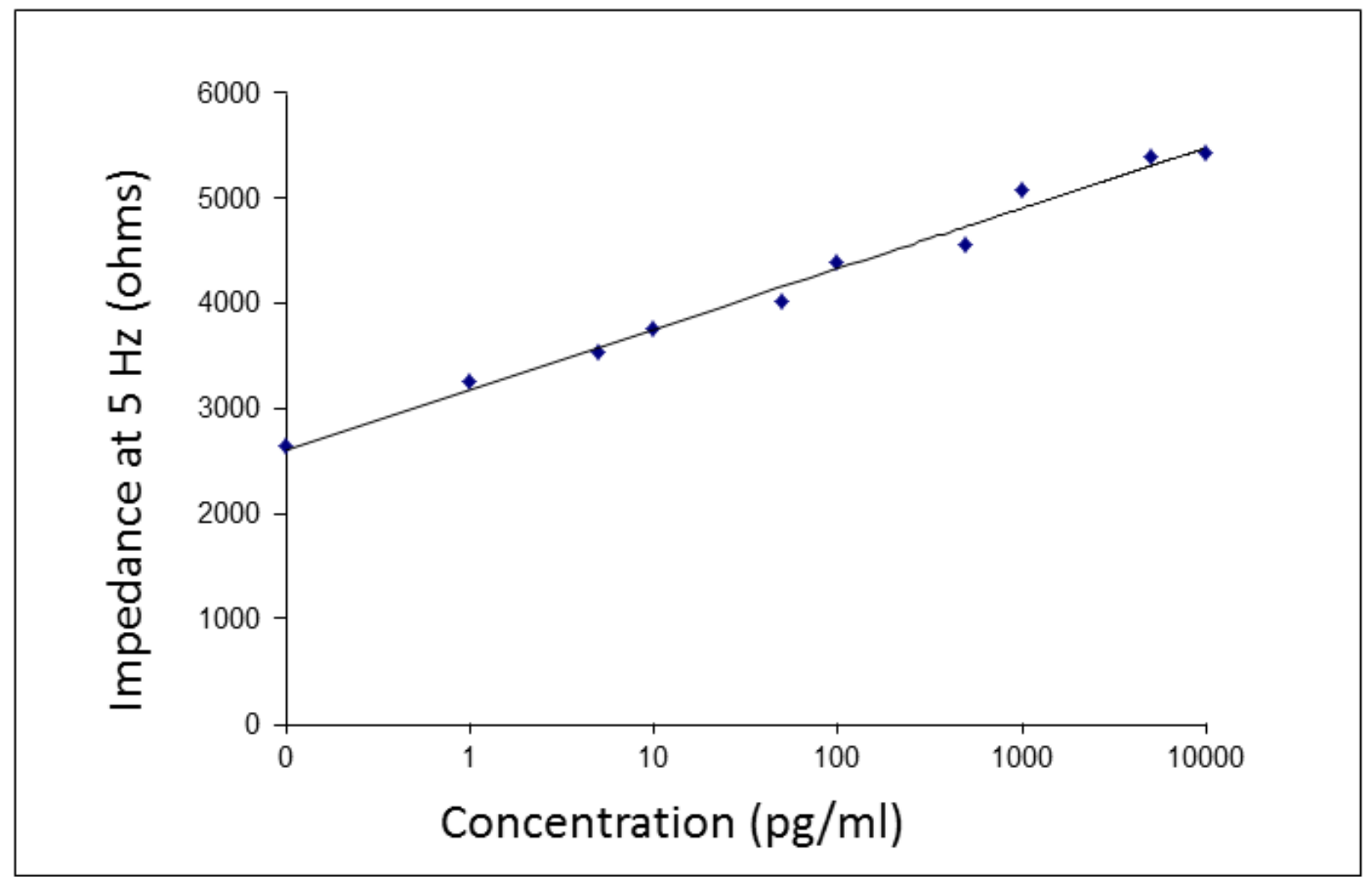

Fig. 8 - Resulting impedance (at $5 \mathrm{~Hz}$ ) concentration

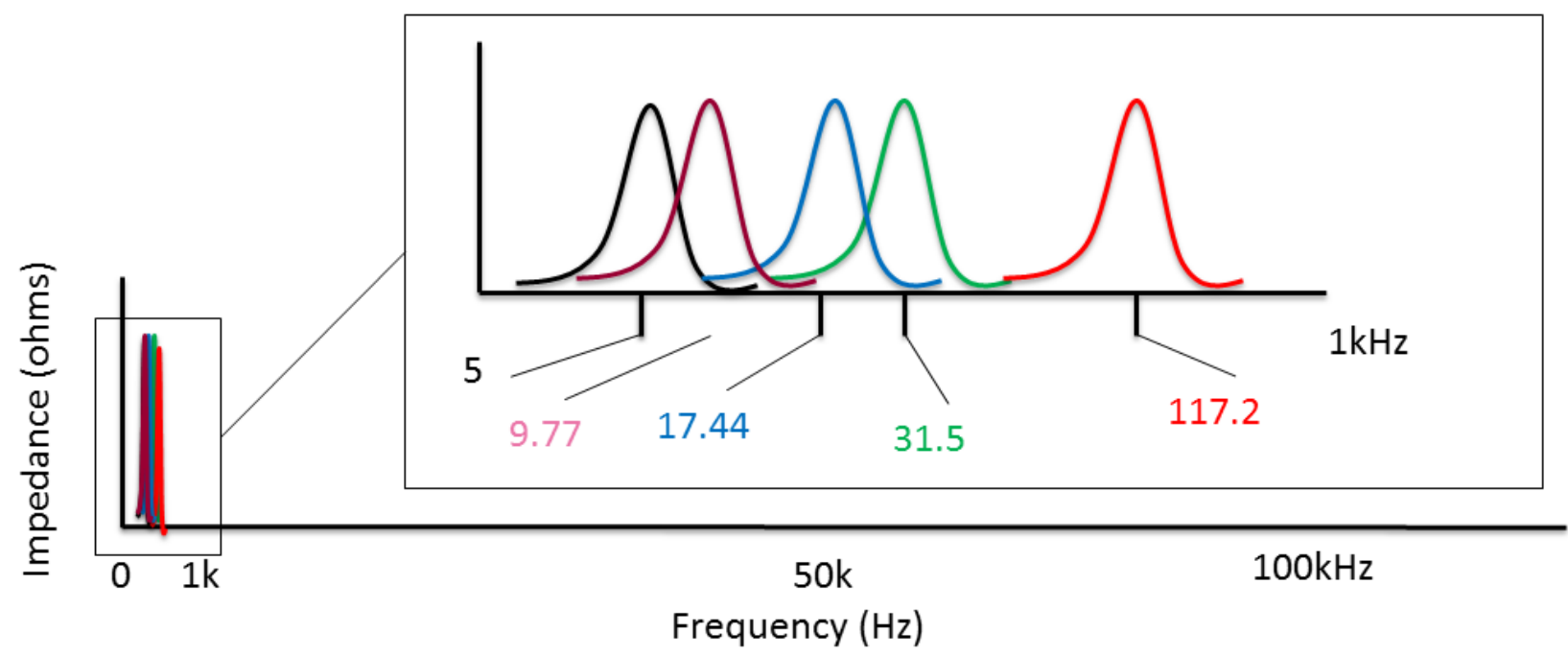

Fig. 9 - Representative impedance spectra of impedance response for biological targets, emphasizing (INSET) where most of the biological "activity" can be monitored, i.e. the low frequency ranges. 
(a)

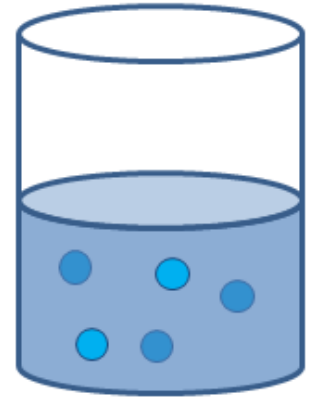

(b)

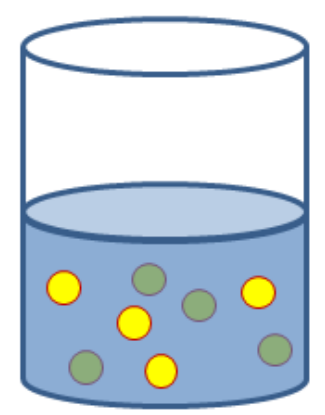

(c)
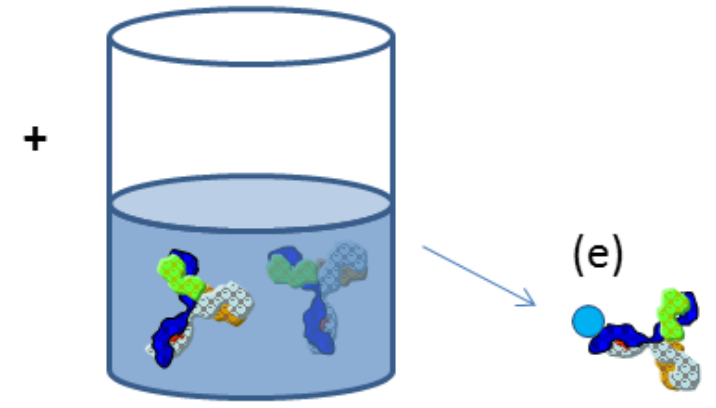

(d)

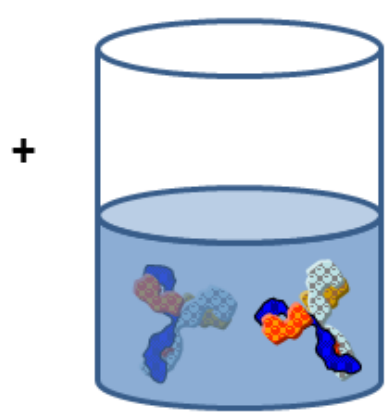

(f) (g)

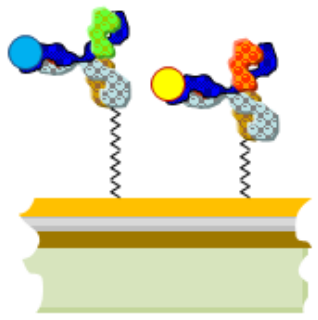

(h)

Fig. 10 - Representative schematic showing possible method for a 2-target system with (a\&b) a solution of (NP's type 1 and 2) being conjugated separately with (c\&d) molecular recognitions elements 1 and 2, producing (e) type 1 NP-MAb and (f) type 2 NP-MAb which can be (g) co-immobilized onto (h) the sensor surface. 


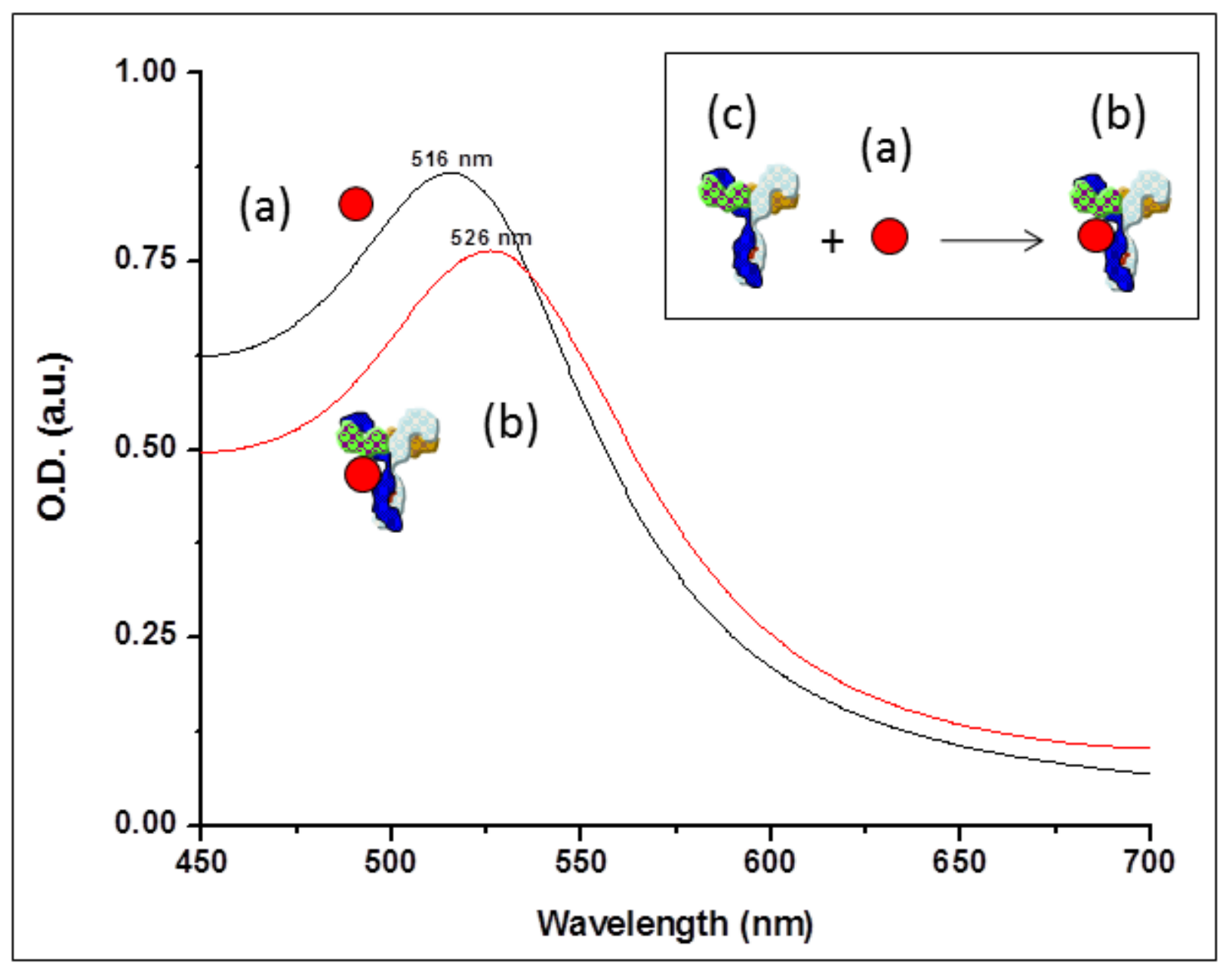

Fig. 11 - Verification of successful conjugation using UV/VIS spectra noting red-shift from 516 to $526 \mathrm{~nm}$ of (c) antibody to (a) AuNP resulting in (b) a conjugated pair using UV/VIS spectra noting shift.
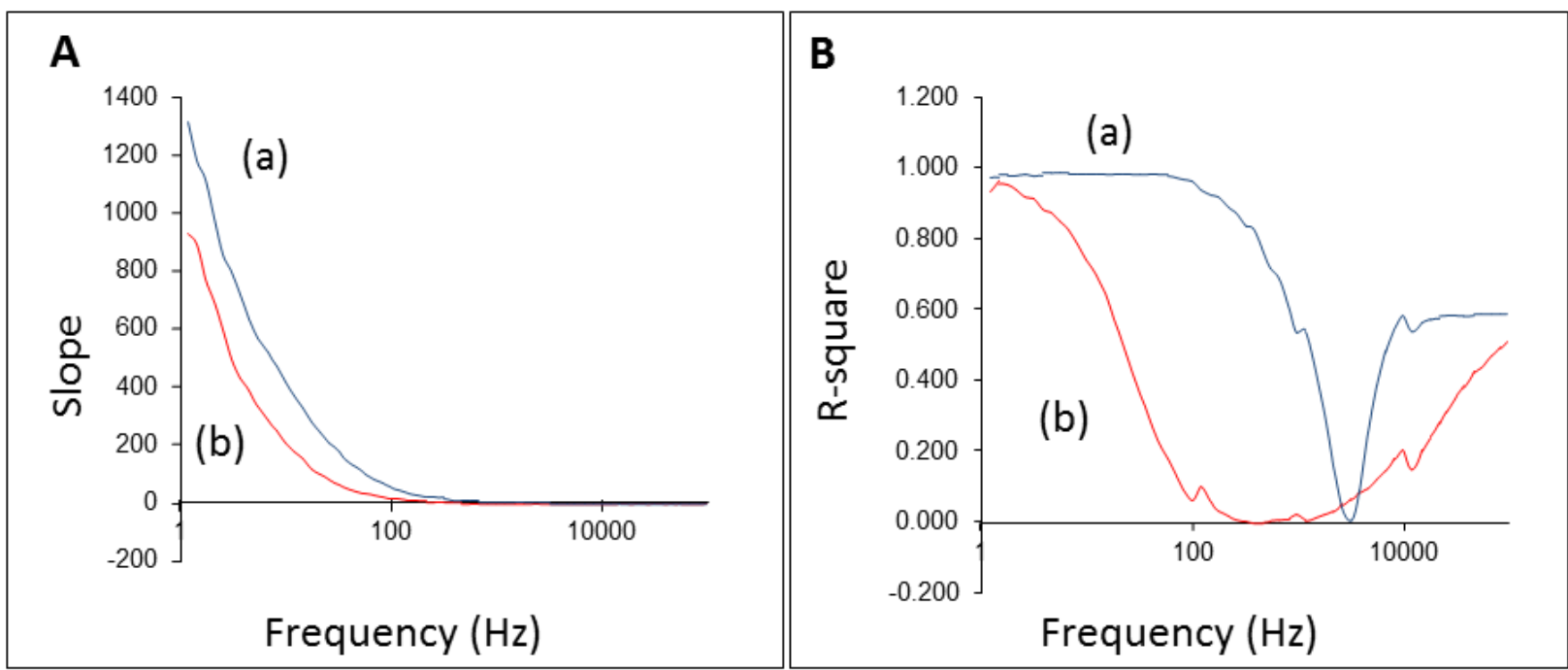
Fig. 12 - A: slope versus frequency plot for (a) unturned and (b) tuned AuNP-MAb pair and B: R-square versus frequency plot for (a) untuned and (b) tuned AuNP-MAb pair showing degree of tuning.

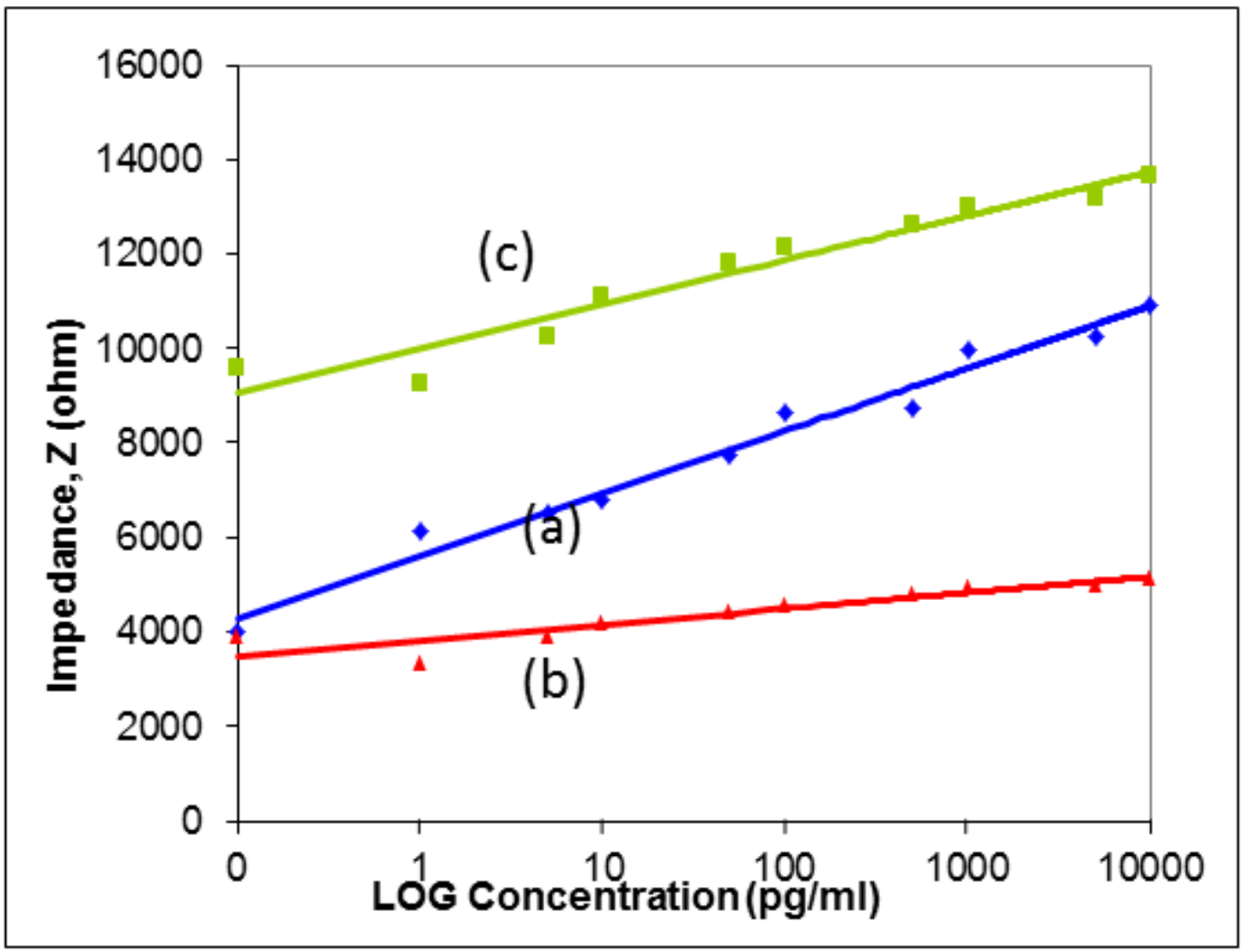

Fig. 13 - Impedance versus concentration plot at (a) $5 \mathrm{~Hz}$ for unconjugated antibody, (b) $5 \mathrm{~Hz}$ for AuNP conjugated antibody and (c) new optimal frequency of $1.43 \mathrm{~Hz}$ AuNP conjugated antibody.
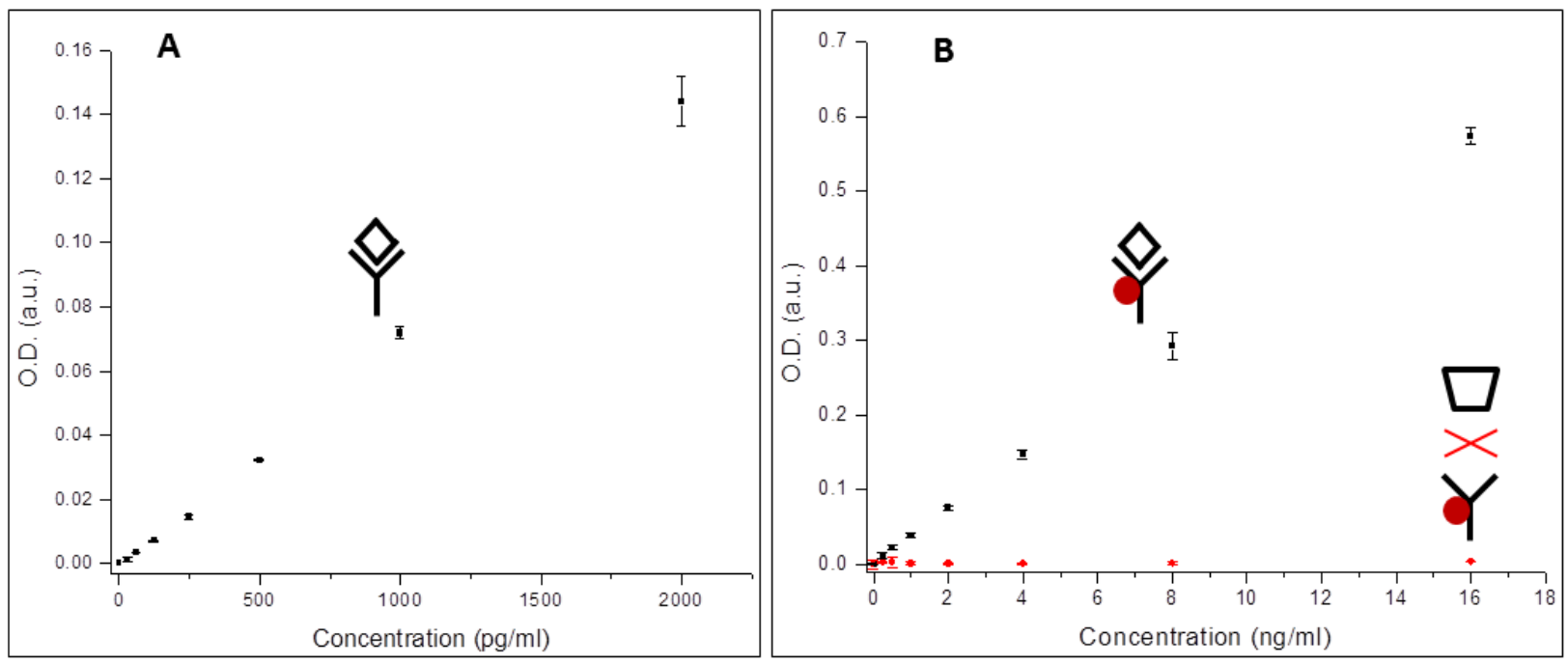
Fig. 14 - ELISA verification of A: AuNP conjugated MAb functionality with untuned MAb function against IL-12 compared to B: tuned conjugate against IL-12 (black squares) and against TNF-a (red triangles) showing high specificity remains for IL-12 and little to no cross reactivity to TNF-a.

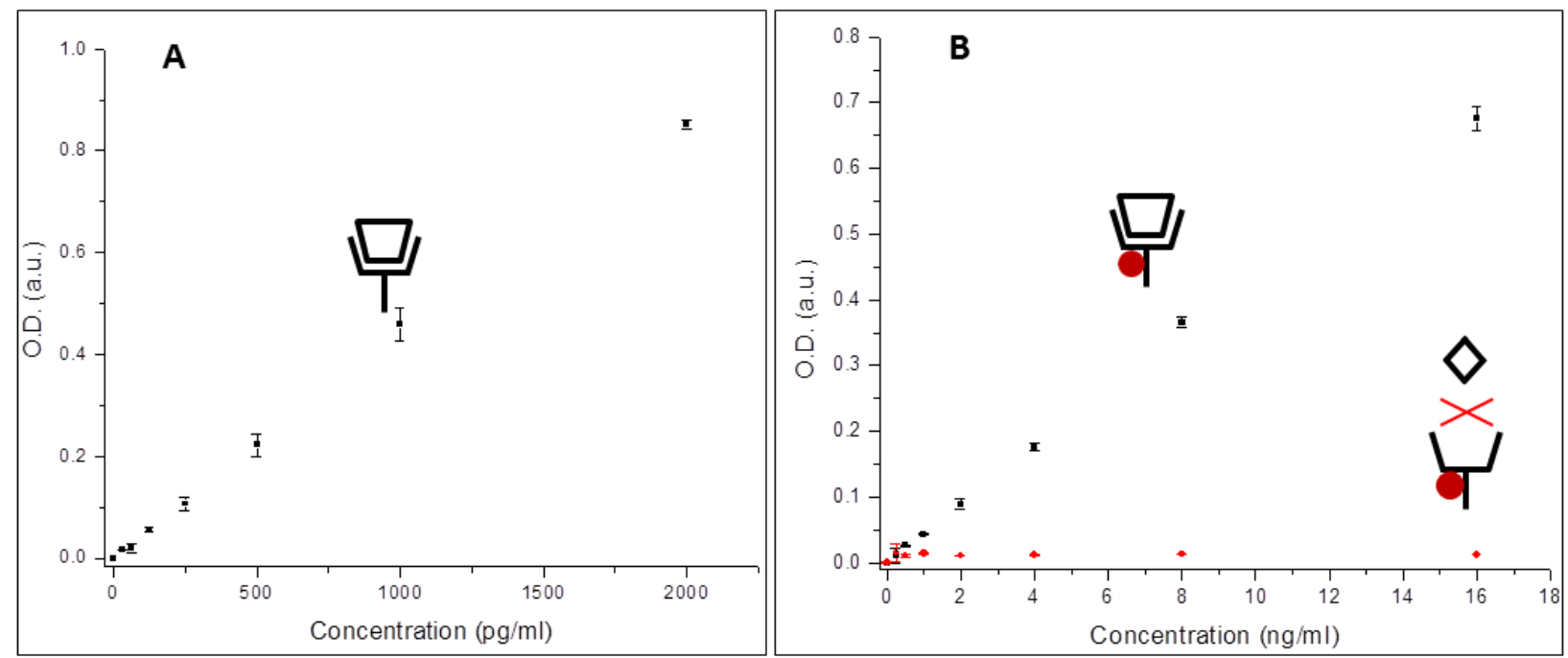

Fig. 15 - ELISA verification of A: AuNP conjugated MAb functionality with untuned MAb function against TNF-a compared to B: tuned conjugate against TNF-a (black squares) and against IL-12 (red triangles) showing high specificity remains for TNF-a and little to no cross reactivity to IL-12. 